\title{
American-type basket option pricing: a simple two-dimensional Partial Differential Equation *
}

\author{
Hamza Hanbali ${ }^{\dagger}$ \\ Dept. Accountancy, Finance and Insurance \\ Email: hamza.hanbali@kuleuven.be
}

KU Leuven

\author{
Daniël Linders \\ Dept. of Mathematics \\ Email: dlinders@illinois.edu \\ University of Illinois
}

October 31, 2019

\begin{abstract}
We consider the pricing of American-type basket derivatives by numerically solving a partial differential equation (PDE). The curse of dimensionality inherent to basket derivative pricing is circumvented by using the theory of comonotonicity. We start with deriving a PDE for the Europeantype comonotonic basket derivative price, together with a unique self-financing hedging strategy. We show how to use the results for the comonotonic market to approximate American-type basket derivative prices for a basket with correlated stocks. Our methodology generates American basket option prices which are in line with the prices obtained via the standard Least-Square Monte Carlo approach. Moreover, the numerical tests illustrate the performance of the proposed method in terms of computation time, and highlight some deficiencies of the standard LSM method.
\end{abstract}

Keywords: Black \& Scholes, basket options, pricing and hedging, comonotonicity, partial differential equations, finite difference method, Least-Squares Monte-Carlo.

\footnotetext{
*Daniël Linders acknowledges the financial support of the Onderzoeksfonds KU Leuven (GOA/13/003). Hamza Hanbali acknowledges the financial support of the AG Chair in Health Insurance at KU Leuven. We are very grateful to Jan Dhaene, Michel Vellekoop and to conference and seminar participants at the University of Amsterdam and KU Leuven for their helpful comments and suggestions.

${ }^{\dagger}$ Corresponding author. Mailing Address: Naamsestraat 69, 3000 Leuven, Belgium.
} 


\section{Introduction}

A European-type basket derivative is a contingent claim written on a basket of stocks, i.e. a weighted average of stock prices. There is a single pay-off at maturity which depends on the realization of the stock prices composing the basket. Stock prices are assumed to be dependent, and therefore a multivariate stock price model is required to price and hedge basket derivatives. Realistic stock price models do not allow for closed-form solutions for European-type basket derivative prices. Instead, one has to use analytical approximations or numerical methods to derive basket derivative prices and to deal with the curse of dimensionality inherent to basket derivative pricing; see e.g. Krekel et al. (2006), Linders and Stassen (2016), Escobar et al. (2016) and Caldana et al. (2016), among others.

American-type basket derivatives are offering early exercise possibilities and are therefore adding another layer of complexity to the pricing problem. The Least-Squares Monte Carlo (LSM) method proposed in Longstaff and Schwarts (2001) is currently one of the preferred methodologies for pricing American-type derivatives. The LSM method provides accurate and efficient pricing for low dimensional basket derivatives, but can be time-consuming if the basket size is large. Moreover, the primary focus of the LSM method is on generating accurate prices. Therefore, there is room for improvement in determining the exercise region and the delta of American options. For example, Wang and Caflisch (2010) provide a modified Least Squares Monte Carlo method which is able to determine the exercise region and the Greeks.

Basket derivative prices with early exercise features can also be determined as the solution of a Partial Differential Equation (PDE). The PDE approach has already be proven useful for determining accurate prices for American-type derivatives. In addition, it also allows to extract the exercise regions and the deltas of the derivative. However, a major drawback of the PDE approach is its curse of dimensionality. Indeed, when the basket dimension is large, pricing basket derivatives by solving a PDE becomes unstable and sometimes even impossible.

The present paper proposes a $P D E$-based approach to approximate prices of American-type basket derivatives. We show that our method is fast and efficient, regardless of the basket size and can be used to price a wide range of exotic basket derivatives. We cope with the curse of dimensionality of the PDE approach by employing the concept of comonotonicity. Comonotonicity describes an extreme market situation where only one stochastic factor drives the stock prices composing the basket. Therefore, the initial multi-dimensional problem is reduced to a univariate problem, and the PDE approach can be used to derive basket derivative prices. Assuming a comonotonic dependence structure solves the dimensionality issue, but does not lead to realistic basket derivative prices. Therefore, we transform the stock volatilities in an appropriate way such that the basket with transformed volatilities, but with a comonotonic dependence structure is a close approximation for the real basket. As a result, basket derivative prices in the comonotonic setting are close to the real basket derivative prices.

The idea of using comonotonicity together with a transformation of the marginal volatilities has been proven useful in solving problems in the area of Asian option pricing, multi-asset derivative pricing, risk management and portfolio selection; see e.g. Dhaene et al. (2002a b) for an overview. For example, various papers have shown that comonotonic pricing formulas with adjusted marginal volatilities can be used to derive accurate closed-form approximations for basket and Asian options; see e.g. Simon et al. (2000), Deelstra et al. (2004), Deelstra et al. (2008), Vyncke et al. (2004), and Linders and Stassen (2016). Moreover, one can prove that under appropriate conditions for the pay-off function, the comonotonic pricing formulas can be employed to determine upper and lower bounds for basket derivative prices; see e.g. Kaas et al. (2000), Dhaene et al. (2000), Dhaene et al. (2002a), Chen et al. (2008) and Chen et al. (2015). The above-mentioned papers deal with pricing European-type options in a risk-neutral valuation context. 
These methodologies focus on approximating the distribution of the basket at the maturity date. Since American-type option pricing additionally requires information about the future paths of all components in the basket, these methodologies cannot be easily extended to American-type basket derivatives. To the best of our knowledge, our paper is the first to use the comonotonicity technique in a PDE framework, such that we can price both European-type as well as American-type basket derivatives.

We introduce the multivariate Black \& Scholes market in Section 2, The stock prices are assumed to be correlated. We show that the theory of comonotonicity can be used to obtain upper and lower bounds for the basket derivative price. In Section 3 we show how to price a basket derivative in a comonotonic market. We derive a partial differential equation (PDE) for the price of a European-type comonotonic basket derivative and provide an explicit expression for the solution of the PDE as an integration over all future states of the Brownian motion driving the comonotonic market. Furthermore, we show that a unique delta-hedging strategy exists for a comonotonic European-type basket derivative. This hedging strategy consists of taking positions in the risk-free bank account and the comonotonic basket. In Section 4, we introduce a simple, yet efficient, finite difference scheme with its corresponding stability criteria to numerically solve the comonotonic PDE for the lower bound, which provides close approximations for the actual prices. Pricing American-type derivatives in a comonotonic setting can be done by implementing the early-exercise opportunity in each step of the finite difference method. We show by a numerical example that using the finite difference method, allows to derive prices, hedging ratios and early exercise regions for comonotonic basket derivatives.

A closer approximate basket derivative price can be derived by applying an appropriate transformation on the stock volatilities and assuming a comonotonic market. This technique was developed in Kaas et al. (2000) and first applied in Simon et al. (2000) to price Asian options and later-on in Deelstra et al. (2004) to price basket options. We use this approach in Section 5 to derive prices for American-type put options and compare the efficiency of the proposed finite different scheme against the LSM method. We first express the price of an option with convex payoff on a basket of stocks as a combination of a comonotonic upper bound and a comonotonic lower bound. We can then use the results of the comonotonic market to express both the upper and lower bounds as solutions of the same two-dimensional PDE, differing only in the initial condition. We show that our proposed method provides accurate prices for American put options. The calculation time is much faster (up to a factor 30) than the LSM method. Moreover, we show that increasing the basket size can have a dramatic effect on the computation time of the LSM method, while our methodology remains fast and accurate.

\section{Basket options in the multivariate Black \& Scholes model}

\subsection{The multivariate Black \& Scholes model}

Consider a market with $n$ non-dividend paying stocks, labeled from 1 to $n$. The price of stock $i$ at time $t \geq 0$ is denoted by $S_{i}(t)$. We assume that the stock price dynamics under the real-world probability measure $\mathbb{P}$ can be described as follows:

$$
S_{i}(t)=S_{i}(0)+\int_{0}^{t} \mu_{i} S_{i}(s) \mathrm{d} s+\int_{0}^{t} \sigma_{i} S_{i}(s) \mathrm{d} B_{i}(s), \text { for } t>0 \text { and } i=1,2, \ldots, n,
$$

where $\underline{B}(t)=\left(B_{1}(t), B_{2}(t), \ldots, B_{n}(t)\right)$ and $\{\underline{B}(t) \mid t \geq 0\}$ is a correlated $n$-dimensional Brownian motion, with $\mathbb{E}\left[\mathrm{d} B_{i}(t) \mathrm{d} B_{j}(t)\right]=\rho_{i, j} \mathrm{~d} t$, defined on a common probability space, equipped with a filtration which records the 'past behavior' of the multivariate stock price process. The market is assumed to satisfy the common requirements of an efficient and frictionless market. 
We assume the risk-free rate $r$ to be deterministic and constant over time. We denote by $D(t)$ the time- $t$ value of the risk-free bank account and its dynamics can be described using the following differential equation:

$$
\frac{\mathrm{d} D(t)}{D(t)}=r \mathrm{~d} t
$$

We denote by $S(t)$ the time-t price of the stock basket, which is expressed as a weighted average of the $n$ individual stocks:

$$
S(t)=w_{1} S_{1}(t)+w_{2} S_{2}(t)+\ldots+w_{n} S_{n}(t)
$$

where the weights $w_{i}$, for $i=1, \ldots, n$, are positive constants. The subsequent results can be adapted to account for positive time-varying weights without loss of generality.

\subsection{Pricing basket options in the multivariate Black \& Scholes model}

A basket derivative with maturity $T$ and pay-off function $H$ is a contingent claim with pay-off determined by the function $H$ and the sample path of the basket. For European-type derivatives, the pay-off at maturity is given by $H(S(T))$, and only depends on the time- $T$ basket price. At time $t$, the basket spot price $S(t)$ together with the stock prices $S_{i}(t)$ are assumed to be known and the arbitrage-free price of the basket derivative is denoted by $V\left(t, S_{1}(t), S_{2}(t), \ldots, S_{n}(t)\right)$. In order to simplify the notation, we sometimes write $V\left(t, S_{1}, S_{2}, \ldots, S_{n}\right)$ or $V$, if no confusion is possible.

Consider an $n$-dimensional basket option with time- $t$ price denoted by $V\left(t, S_{1}, S_{2}, \ldots, S_{n}\right)$, where $S_{1}, S_{2}, \ldots, S_{n}$ are the basket components. Then one can prove that in a Black \& Scholes setting, the price $V$ of a European-type derivative satisfies the following partial differential equation (PDE):

$$
\frac{\partial V}{\partial t}+\frac{1}{2} \sum_{i=1}^{n} \sum_{j=1}^{n} \sigma_{i} \sigma_{j} \rho_{i, j} w_{i} w_{j} S_{i} S_{j} \frac{\partial^{2} V}{\partial S_{i} \partial S_{j}}+r \sum_{i=1}^{n} S_{i} \frac{\partial V}{\partial S_{i}}-r V=0
$$

where the $w_{i}$ are constant and positive basket weights and $\left(\sigma_{i} \sigma_{j} \rho_{i, j}\right)_{1 \leq i, j \leq n}$ is the variance-covariance matrix of the stock prices. Taking into account appropriate boundary and final conditions, the time- $t$ price of the basket derivative is the solution of this equation. However, the analytical solution requires an $n$-dimensional integration and evaluating such an integral is impossible for high dimensions. Instead, one can opt to use finite difference methods to numerically solve the differential equation 2.3. This, however, requires a time grid and an $n$-dimensional stock price grid, resulting in a procedure which is too slow to be used for pricing and calibration purposes, especially for high basket dimensions. As a result, the problem of basket option pricing using PDE methods remains an open problem.

The main advantage of PDE methods over other existing pricing methods, such as risk-neutral valuation, is the possibility to incorporate path dependency and early exercise features and to determine the exercise region for American-type options. In the one-dimensional case, standard numerical methods such as finite difference can be employed to numerically price an American-type option in the PDE framework; see e.g. Haentjens and In’t Hout (2015) and Witelski and Bowen (2003). For higher dimensions, however, such numerical schemes become instable and cannot provide accurate results. These issues are predominantly caused by the cross-derivative terms in the multivariate PDE (2.3). Several numerical methods exist to deal with the cross-derivative terms, resulting in complex approximate prices for basket derivatives; see e.g. Reisinger and Wittum (2007), Leentvaar and Oosterlee (2008), Clift and Forsyth (2008), Düring and Heuer (2015) and Company et al. (2016). 


\subsection{An upper bound for basket derivative prices with convex payoff}

A possible solution to tackle the problem of pricing basket derivatives, consists in deriving upper and lower bounds for the derivative price. An upper bound for the basket derivative price is obtained by making the basket 'more volatile'. We are considering the same stocks as in the original basket, but we assume they all move in the same direction. In case the payoff function of the derivative is a convex function, employing an extreme positive dependence structure leads to an upper bound for the basket derivative price.

We construct an artificia ${ }^{1}$ financial market where all stocks behave in a comonotonic way. In this comonotonic market, $n$ stocks are traded and the price of stock $i$ at time $t$ is denoted by $S_{i}^{c}(t)$. The dynamics of the $n$ traded stocks is described by the following stochastic integral equation (SIE):

$$
S_{i}^{c}(t)=S_{i}(0)+\int_{0}^{t} \mu_{i} S_{i}^{c}(s) \mathrm{d} s+\int_{0}^{t} \sigma_{i} S_{i}^{c}(s) \mathrm{d} B(s), \text { for } t>0 \text { and } i=1,2, \ldots, n,
$$

where $B=\{B(t) \mid t \geq 0\}$ is a standard Brownian motion and $S_{i}(0)$, for $i=1,2, \ldots, n$, are the time- 0 prices of the basket components.

The stocks in the comonotonic market are equal, in distribution, to the original stocks:

$$
S_{i}^{c}(t) \stackrel{d}{=} S_{i}(t), \text { for } i=1,2, \ldots, n \text { and } t>0 .
$$

However, the price vector $\left(S_{1}^{c}(t), S_{2}^{c}(t), \ldots, S_{n}^{c}(t)\right)$ has a comonotonic copula and the correlation between the $\log$ returns is always equal to one. The price of the comonotonic basket at time $t$ is denoted by $S^{c}(t)$ and:

$$
S^{c}(t)=w_{1} S_{1}^{c}(t)+w_{2} S_{2}^{c}(t)+\ldots+w_{n} S_{n}^{c}(t) .
$$

Consider a derivative with convex pay-off function $H$ and maturity $T$, but written on the comonotonic basket $S^{c}(T)$. The time-t price of this comonotonic basket derivative is unambiguously determined by the basket spot $S^{c}(t)$. In a general, non-comonotonic market, this is not the case: knowledge about the realization $S(t)$ is not sufficient to determine $V\left(t, S_{1}, \ldots, S_{n}\right)$. Therefore, we can denote the comonotonic time- $t$ price by $V^{c}\left(t, S^{c}(t)\right)$. Moreover, Kaas et al. (2000) proved that any time $t$, the comonotonic basket option price $V^{c}\left(t, S^{c}(t)\right)$ is an upper bound for the original price $V\left(t, S_{1}, \ldots, S_{n}\right)$.

The main advantage of working in a comonotonic market lies in the dimension reduction of the problem it produces. Indeed, basket derivative pricing in the comonotonic market is essentially a onedimensional problem. The main disadvantage of the comonotonic market is that it does not provide realistic basket derivative prices. In the next subsection we consider a comonotonic market which does allow for realistic basket derivative prices by employing an appropriate transformation of the marginal distributions.

\subsection{A lower bound for the basket derivative price with convex payoff}

Consider again an artificial comonotonic market with $n$ stock prices labelled $S_{i}^{l}$, for $i=1, \ldots, n$. The dynamics of the time- $t$ prices of the $S_{i}^{l}$,s are given by

$$
S_{i}^{l}(t)=S_{i}(0)+\int_{0}^{t} \mu_{i} S_{i}^{l}(s) \mathrm{d} s+\int_{0}^{t} \nu_{i} \sigma_{i} S_{i}^{l}(s) \mathrm{d} B(s), \text { for } t>0 \text { and } i=1,2, \ldots, n,
$$

\footnotetext{
${ }^{1}$ we use the term 'artificial' to emphasize that the market described by the SIE's 2.4 is a fictitious market and does not intend to describe the real market situation.
} 
where $0 \leq \nu_{i} \leq 1$, for $i=1,2, \ldots, n$. The time- $t$ stock prices have now different distributions than the real stock prices. To be more precise, the artificial stock price processes $S_{i}^{l}, i=1,2, \ldots, n$ are less volatile compared to the real stock price processes $S_{i}$, because $0<\nu_{i}<1$. Indeed, it is straightforward to prove that

$$
\operatorname{Var}\left[\frac{S_{i}^{l}(t)}{\mathbb{E}\left[S_{i}^{l}(t)\right]} \mid \mathcal{F}_{0}\right] \leq \operatorname{Var}\left[\frac{S_{i}(t)}{\mathbb{E}\left[S_{i}\right]} \mid \mathcal{F}_{0}\right] .
$$

Since $\nu_{i}>0$, the time- $t$ stock price vector $\left(S_{1}^{l}, \ldots, S_{n}^{l}\right)$ has again a comonotonic copula.

The corresponding basket is denoted by $S^{l}(t)$ and

$$
S^{l}(t)=w_{1} S_{1}^{l}(t)+w_{2} S_{2}^{l}(t)+\ldots+w_{n} S_{n}^{l}(t) .
$$

The distribution of $S^{l}(t)$ depends on the choice of the factors $\nu_{i}, i=1,2, \ldots, n$. The time- $t$ price of a basket derivative with convex payoff function $H$, written on the basket $S^{l}$ is denoted by $V^{l}\left(t, S^{l}\right)$. The lemma below provides sufficient conditions for the factors $\nu_{i}, i=1,2, \ldots, n$ such that the basket derivative price $V^{l}\left(t, S^{l}\right)$ will be a lower bound for the basket derivative price $V(t, S)$.

Lemma 2.1 Assume the factors $\nu_{i}, i=1,2, \ldots, n$ in the stock price model (2.7) are given by

$$
\nu_{i}=\frac{\sum_{j=1}^{n} \beta_{j} \rho_{i, j} \sigma_{j}}{\sqrt{\sum_{j=1}^{n} \sum_{k=1}^{n} \beta_{j} \beta_{k} \rho_{j, k} \sigma_{j} \sigma_{k}}} \text {, for } i=1,2, \ldots, n,
$$

with $\beta_{j}>0$ for $j=1,2, \ldots, n$. Then, there always exists a choice for the $\beta_{j}>0, j=1,2, \ldots, n$, such that $0 \leq \nu_{i} \leq 1, i=1,2, \ldots, n$ and

$$
V^{l}\left(t, S^{l}\right) \leq V(t, S)
$$

For suggestions on the choice for the $\beta_{j}$ parameters and a proof of Lemma 2.1, we refer to Kaas et al. (2000), Deelstra et al. (2004) and Linders and Stassen (2016).

To conclude, we can derive an upper and a lower bound for the real derivative price $V\left(t, S_{1}, S_{2}, \ldots, S_{n}\right)$ :

$$
V^{l}\left(t, S^{l}\right) \leq V\left(t, S_{1}, S_{2}, \ldots, S_{n}\right) \leq V^{c}\left(t, S^{c}\right) .
$$

Moreover, both the upper and lower bound can be determined in a comonotonic market. In the following section, we search for the basket derivative prices $V^{c}\left(t, S^{c}\right)$ and $V^{l}\left(t, S^{l}\right)$, and we show that the corresponding PDE's reduce to two-dimensional PDE's. As a result, the closed-form solutions consist of single integrations over Gaussian densities and only a one-dimensional stock price grid is needed for each PDE if a finite difference method is employed. Note that the dynamics of the baskets $S^{l}$ and $S^{c}$ differ only in the marginal volatilities. Therefore, we exploit this similarity and we derive our results for the upper bound only.

\section{The comonotonic basket derivative}

\subsection{Dynamics of the comonotonic basket}

We find from Expressions (2.4) and (2.6) that the time- $t$ stock prices $S_{i}^{c}(t), i=1,2, \ldots, n$ and the time- $t$ basket price $S^{c}(t)$ can be expressed as functions of $t$ and the Brownian motion $B$. Moreover, since the functions $f_{i}: \mathbb{R} \rightarrow \mathbb{R}$, for $i=1,2, \ldots, n$, defined by

$$
f_{i}(x)=w_{i} S_{i}(0) \mathrm{e}^{\left(\mu_{i}-\frac{1}{2} \sigma_{i}^{2}\right) t+\sigma_{i} x}
$$


are strictly increasing, the function

$$
f(x)=\sum_{i=1}^{n} f_{i}(x)
$$

is also strictly increasing. Hence, for any time partitioning $0 \leq t_{1}<\ldots<t_{m} \leq T$ and real numbers $x_{1}, x_{2}, \ldots, x_{m}$, where $m$ is an integer, the sets $\left\{\omega \in \Omega \mid S^{c}\left(t_{1}\right)<x_{1}, S^{c}\left(t_{2}\right)<x_{2}, \ldots, S^{c}\left(t_{m}\right)<x_{m}\right\}$ and $\left\{\omega \in \Omega \mid B\left(t_{1}\right)<f^{-1}\left(x_{1}\right), B\left(t_{2}\right)<f^{-1}\left(x_{2}\right), \ldots, B\left(t_{m}\right)<f^{-1}\left(x_{m}\right)\right\}$ are equal. Therefore, the natural filtration of the Brownian motion and the filtration generated by the comonotonic basket price coincide. We say that the comonotonic market is driven by the single random source $B$.

In the following lemma we show that the comonotonic basket $S^{c}=\left\{S^{c}(t) \mid t \geq 0\right\}$ follows an Ito process with time-dependent drift and volatility.

Lemma 3.1 Consider the comonotonic market described in (2.4). The stochastic integral equation of the comonotonic basket $S^{c}$ defined in (2.6) is given by:

$$
S^{c}(t)=S(0)+\int_{0}^{t} \mu^{c}(s) d s+\int_{0}^{t} \sigma^{c}(s) d B(s)
$$

where

$$
\mu^{c}(s)=\sum_{i=1}^{n} \mu_{i} w_{i} S_{i}^{c}(s) \text { and } \sigma^{c}(s)=\sum_{i=1}^{n} \sigma_{i} w_{i} S_{i}^{c}(s)
$$

Proof. Since $S^{c}(t)=\sum_{i=1}^{n} w_{i} S_{i}^{c}(t)$, the stochastic integral equation 3.2) follows directly from 2.4 using an inversion of sums and integrals.

Note that we can also use the conventional expression

$$
\mathrm{d} S^{c}(t)=\mu^{c}(t) \mathrm{d} t+\sigma^{c}(t) \mathrm{d} B(t),
$$

to describe the dynamics of the comonotonic basket, instead of the SIE (3.2).

The comonotonic market described above is assumed to be arbitrage-free. This no-arbitrage condition is equivalent with the existence of a parameter $\lambda \in \mathbb{R}$ satisfying

$$
\lambda=\frac{\mu_{i}-r}{\sigma_{i}}, \text { for } i=1,2, \ldots, n,
$$

which is called the market price of risk; it expresses the reward one obtains for picking up a unit of volatility. Indeed, it is the increase in the expected excess return $\mu_{i}-r$ per unit of volatility. In the comonotonic market, the market price of risk is the same for each stock because the volatility of each stock is driven by the same Brownian motion and hence a unit of volatility of stock $i$ is essentially the same as a unit of volatility of any other stock $j$. For a detailed discussion on no-arbitrage conditions in the multivariate Black \& Scholes model, we refer to Björk (1998), Dhaene et al. (2014) and Dhaene et al. (2018).

Define the process $B_{\lambda}=\left\{B_{\lambda}(t) \mid t \geq 0\right\}$ as follows:

$$
\mathrm{d} B_{\lambda}(t)=\mathrm{d} B(t)+\lambda \mathrm{d} t .
$$

Hence, $B_{\lambda}$ is a Brownian motion with drift, where the drift parameter is equal to the market price of risk of the comonotonic market. On the one hand, we can express the marginal stock prices $S_{i}^{c}(t)$ in terms of 
the process $B_{\lambda}$ by plugging (3.5) in Expression (3.4) and using the relation $r=\mu_{i}-\lambda \sigma_{i}$. The SDE for $S^{c}$ can be expressed as follows:

$$
\mathrm{d} S^{c}(t)=r S^{c}(t) \mathrm{d} t+\sigma^{c}(t) \mathrm{d} B_{\lambda}(t),
$$

and the basket $S^{c}$ can be expressed as follows

$$
S^{c}(t)=\sum_{i=1}^{n} w_{i} S_{i}(0) \mathrm{e}^{\left(r-\frac{1}{2} \sigma_{i}^{2}\right) t+\sigma_{i} B_{\lambda}(t)} .
$$

We also find that

$$
\mu^{c}(t)-\sigma^{c}(t) \lambda=r S^{c}(t)
$$

Girsanov's theorem states that there exists a probability measure $\mathbb{Q}$ such that the stochastic process $B_{\lambda}$ is a Brownian motion under this new probability measure. Since $B_{\lambda}$ has drift equal to the market price of risk, the probability measure $\mathbb{Q}$ corresponds with the risk-neutral pricing measure. Note that under the probability measure $\mathbb{P}$, this process is a Brownian motion with drift, whereas under the risk-neutral measure $\mathbb{Q}$, the process $B_{\lambda}$ is a standard Brownian motion. In the remainder of the paper, we use the SDE (3.6) for the dynamics of the comonotonic basket but we work under the objective probability measure $\mathbb{P}$. This means that the process $B_{\lambda}$ is a Brownian motion with drift $\lambda$.

\subsection{The PDE for the basket derivative price}

The comonotonic market is driven by a single random source: the process $B_{\lambda}$. The realization of $B_{\lambda}$ at time $t$ unambiguously determines the realizations of $S^{c}(t)$ and $S_{i}^{c}(t)$. As a result, the price of the basket derivative can be expressed as a function of the time $t$ and the realization $B_{\lambda}$ of the random source. The time- $t$ comonotonic basket derivative price is then written using an alternative expression for the function $V_{\lambda}^{c}$, which is defined as follows:

$$
V_{\lambda}^{c}\left(t, B_{\lambda}\right)=V^{c}\left(t, \sum_{i=1}^{n} w_{i} S_{i}(0) \mathrm{e}^{\left(r-\frac{1}{2} \sigma_{i}^{2}\right) t+\sigma_{i} B_{\lambda}}\right) .
$$

Note that we use the same $t$ for $V^{c}\left(t, S^{c}\right)$ and $V_{\lambda}^{c}\left(t, B_{\lambda}\right)$ by abuse of notation only. When writing $V^{c}\left(t, S^{c}\right)$, it is implicitly assumed that $S^{c}$ is constant when the time varies, whereas in $V_{\lambda}^{c}\left(t, B_{\lambda}\right)$ the basket $S^{c}$ is time varying if $t$ varies and $B_{\lambda}$ remains constant. Therefore, we have:

$$
\frac{\partial V_{\lambda}^{c}}{\partial t}\left(t, B_{\lambda}\right)=\frac{\partial V^{c}}{\partial t}\left(t, S^{c}\right)+\frac{\partial V^{c}}{\partial S^{c}}\left(t, S^{c}\right) \frac{\partial S^{c}}{\partial t}\left(t, B_{\lambda}\right) .
$$

A trading strategy is denoted by the stochastic process $\pi=\left\{\left(\pi_{1}(t), \pi_{2}(t)\right) \mid t \geq 0\right\}$, where $\pi_{1}(t)$ and $\pi_{2}(t)$ are the number of units invested at time $t$ in the comonotonic basket and the risk-free bank account, respectively, and satisfy the standard assumption of $\mathbb{P}$-a.s. square integrability on $[0, T]$. The time- $t$ value of the strategy $\pi$ is denoted by $\Pi(t)$ and

$$
\Pi(t)=\pi_{1}(t) S^{c}(t)+\pi_{2}(t) D(t) .
$$

A trading strategy is said to be self-financing if one can inject or withdraw money from the investment portfolio only at the beginning and the end date of the strategy. All other purchases in one asset class have to be financed by selling positions in the other asset class. 


\subsubsection{European-type basket derivative}

Assume the basket derivative is of European type, i.e. it has a pay-off at maturity only. We derive the partial differential equation for the price $V_{\lambda}^{c}$ in Theorem 3.1, whose proof can be found in the appendix. We also show that there exists a unique self-financing portfolio which replicates the comonotonic basket derivative price. Since the comonotonic basket derivative $S^{c}$ is an Ito process, the proof follows the same spirit as the classical Black \& Scholes PDE proof; see e.g. Theorem 3.1.1 in Musiela and Rutkowski (2005).

Theorem 3.1 The derivative price $V_{\lambda}^{c}$ with pay-off at time $T$ given in (3.9) satisfies the following backward partial differential equation:

$$
\frac{\partial V_{\lambda}^{c}}{\partial t}+\frac{1}{2} \frac{\partial^{2} V_{\lambda}^{c}}{\partial B_{\lambda}^{2}}-r V_{\lambda}^{c}=0
$$

where the final condition is given by

$$
V_{\lambda}^{c}\left(T, B_{\lambda}\right)=H\left(\sum_{i=1}^{n} w_{i} S_{i}(0) e^{\left(r-\frac{1}{2} \sigma_{i}^{2}\right) T+\sigma_{i} B_{\lambda}}\right) .
$$

Furthermore, the unique, self-financing, replicating strategy $\pi=\left\{\left(\pi_{1}(t), \pi_{2}(t)\right) \mid t \geq 0\right\}$ of this derivative is given by:

$$
\begin{aligned}
\pi_{1}(t) & =\frac{1}{\sigma^{c}} \frac{\partial V^{c}}{\partial B_{\lambda}} \\
\pi_{2}(t) & =\frac{1}{D(t)}\left(V^{c}\left(t, B_{\lambda}\right)-\pi_{1}(t) S^{c}(t)\right) .
\end{aligned}
$$

Equation (3.8) is a backward linear parabolic partial differential equation for the comonotonic basket price with constant coefficients. It has only two dimensions, regardless of the number of the basket components. The single dimensionality and the constant coefficients are due to the fact that only one random source is governing the comonotonic market, and allow for an efficient numerical scheme. The backward nature of this equation requires to impose the final condition (3.9), which corresponds to the pay-off at time $T$, and hence, determines the type of the option.

In order to ensure the uniqueness of the solution of the PDE, we must also impose boundary conditions. These conditions are carrying information on the type of the option under consideration. For instance, the boundary conditions for a barrier option have to take into account the set of values of the comonotonic basket for which the option is worthless. Therefore, boundary conditions must be chosen carefully, with a realistic financial interpretation, to reflect the specificities of the basket derivative. We refer to Wilmott (2006), among others, for a discussion on the boundary conditions which are applicable here, with practical aspects on their numerical implementation. We end the comments on Theorem 3.1 with a remark on the assumption of a constant interest rate $r$ and the assumption that we deal with a non-dividend paying basket. In practice, Equation 3.8 can readily be extended to account for timevarying deterministic interest rates and to include dividend yields using similar techniques as in the one-dimensional Black \& Scholes case.

\subsubsection{Continuously sampled Asian-type basket derivatives}

We consider a continuously sampled Asian basket option; a derivative with pay-off function depending on the average basket price on the interval $[0, T]$. The average used in the Asian option is denoted by 
$I(t)$ and is defined as follows:

$$
I(t)=\int_{0}^{t} g\left(u, S^{c}\right) \mathrm{d} u
$$

where the function $g$ determines the type of the agreed-upon averaging, e.g. arithmetic or geometric average. A similar reasoning is applied to Look-back options, for which the function $g$ has to be adapted accordingly. The price of the Asian basket derivative is now also dependent on $I$. Similar calculations as in the proof of Theorem 3.1 lead to the following PDE that the Asian basket derivative price satisfies:

$$
\frac{\partial V_{\lambda}^{c}}{\partial t}+g\left(t, S^{c}\right) \frac{\partial V_{\lambda}^{c}}{\partial I}+\frac{1}{2} \frac{\partial^{2} V_{\lambda}^{c}}{\partial B_{\lambda}^{2}}-r V_{\lambda}^{c}=0 .
$$

Deriving a closed-form solution for the PDE (3.10) is in general not feasible. However, pricing comonotonic Asian basket options can also be performed numerically. Moreover, given its similarity with the PDE for an Asian option in the one-dimensional Black \& Scholes model, one can tackle the problem of solving (3.10) in the same way; see e.g. Wilmott et al. (1994), Zvan et al. (1998) and Wilmott (2006).

\subsection{Solution of the comonotonic PDE}

In Theorem 3.2 stated below, we show that there exists an explicit solution for Equation 3.8 which satisfies the final condition (3.9). The proof first recasts the PDE (3.8) into a one-dimensional heat equation, after which we solve the one-dimensional heat equation in terms of Gaussian densities using similarity reduction techniques. In the last step, we go back to the original problem and find a solution $V_{\lambda}^{c}$ for (3.8); see e.g. Wilmott (2006). Note that the following Theorem enables to derive further results on the distributions of the comonotonic basket, which are given in the Appendix section.

Theorem 3.2 The time-t price of a European-type derivative with pay-off function $H$ at maturity $T$ written on the comonotonic basket $S^{c}$ is denoted by $V_{\lambda}^{c}\left(t, B_{\lambda}\right)$ and is given by:

$$
V_{\lambda}^{c}\left(t, B_{\lambda}\right)=e^{-r(T-t)} \int_{-\infty}^{+\infty} H\left(\sum_{i=1}^{n} w_{i} S_{i}^{c}(t) e^{\left(r-\frac{1}{2} \sigma_{i}^{2}\right)(T-t)+\sigma_{i} y}\right) \phi_{T-t}(y) \mathrm{d} y,
$$

where $S_{i}^{c}(t)$ is the time-t spot price of the comonotonic stock $i$, which is given by

$$
S_{i}^{c}(t)=S_{i}(0) \mathrm{e}^{\left(r-\frac{1}{2} \sigma_{i}^{2}\right) t+\sigma_{i} B_{\lambda}(t)}, \text { for } i=1,2, \ldots, n,
$$

and $\phi_{T-t}$ denotes the density function of a normal distribution with mean 0 and variance $T-t$ :

$$
\phi_{T-t}(y)=\frac{e^{-\frac{y^{2}}{2(T-t)}}}{\sqrt{2 \pi(T-t)}} .
$$

\section{Finite difference scheme: pricing, hedging and exercise region}

\subsection{The comonotonic finite difference method for European-type basket derivatives}

Solving the comonotonic Black \& Scholes PDE given in (3.8) together with the pay-off $H\left(S^{c}(T)\right)$ at maturity yields the value of the European-type derivative at any time $t$ as a one-dimensional integral; see 
(3.11). In this section, we use the explicit finite difference method to numerically obtain a solution; see e.g. Brennan and Schwartz (1977).

Take the time step $\delta t$ and

$$
t_{k}=T-k \delta t, \text { for } k=0,1, \ldots, N .
$$

Take also a grid with step size $\delta B$ for $B_{\lambda}$ :

$$
b_{j}=(j-I) \delta B, j=0,1, \ldots, J .
$$

for some $I$ and $J$. The value for the comonotonic basket derivative price $V_{\lambda}^{c}\left(t_{k}, b_{j}\right)$ of the contract at the grid point $\left(t_{k}, b_{j}\right)$ is denoted by $V_{j}^{k}$. The partial derivatives in $\left(t, B_{\lambda}\right)$ can be approximated as follows:

$$
\begin{aligned}
\frac{\partial V_{\lambda}^{c}}{\partial t} & =\frac{V_{j}^{k}-V_{j}^{k+1}}{\delta t}+\mathcal{O}(\delta t) \\
\frac{\partial^{2} V_{\lambda}^{c}}{\partial B_{\lambda}^{2}} & =\frac{V_{j+1}^{k}-2 V_{j}^{k}+V_{j-1}^{k}}{\delta B^{2}}+\mathcal{O}\left(\delta B^{2}\right) .
\end{aligned}
$$

While the initial condition $V_{j}^{0}$ characterizing the contract follows from the pay-off function:

$$
V_{j}^{0}=H\left(\sum_{i=1}^{n} w_{i} S_{i}(0) \mathrm{e}^{\left(r-\frac{1}{2} \sigma_{i}^{2}\right) T+\sigma_{i} b_{j}}\right),
$$

the basket derivative prices at the other nodes can be derived by using the approximations (4.1) and (4.2) in the comonotonic PDE (3.8). This gives the following explicit expression for $V_{j}^{k+1}$ given the values $V_{j-1}^{k}, V_{j}^{k}$ and $V_{j+1}^{k}$ :

$$
V_{j}^{k+1}=\frac{1}{2} \frac{\delta t}{\delta B^{2}} V_{j-1}^{k}+\left(1-r \delta t-\frac{\delta t}{\delta B^{2}}\right) V_{j}^{k}+\frac{1}{2} \frac{\delta t}{\delta B^{2}} V_{j+1}^{k}+\mathcal{O}\left(\delta t ; \delta B^{2}\right),
$$

for $j=1, \ldots, J-1$ and $k=0,1, \ldots, N-1$. Moreover, one needs to determine the boundary conditions $V_{0}^{k}$ and $V_{J}^{k}$ which depends on the contract specificities.

Similarly to the one-dimensional Black \& Scholes model, this method can be improved using implicit or semi-implicit methods, such as the Crank-Nicolson method; see Crank and Nicolson (1947), Duffy (2004), Wilmott (2006) and Ahmad (2006).

We provide in the following theorem a condition ensuring the stability of the scheme. The proof can be found in the Appendix.

Theorem 4.1 The finite difference scheme (4.4) is stable when the following condition is satisfied:

$$
\delta t \leq \frac{2 \delta B^{2}}{r \delta B^{2}+2}
$$

\subsection{Numerical Illustration: upper and lower bounds for European-type basket put op- tion}

We illustrate the use of the finite difference method derived in the previous section using a numerical example. Consider a European-type put option, written on a four-stock basket with parameters summarized in Table 1. We first approximate the real price of a basket put option with strike $K$ and maturity $T$ using Monte Carlo simulation with 1000000 simulations. The price is denoted by $V^{\text {Sim }}$. 
Next, we determine the upper bound $V^{c}$ and the lower bound $V^{l}$ using the finite difference scheme. For the lower bound $V^{l}$, we use the parameter $\nu_{i}$ which is chosen as:

$$
\nu_{i}=\frac{\sum_{j=1}^{n} w_{j} S_{j}(0) \rho_{i, j} \sigma_{j}}{\sqrt{\sum_{j=1}^{n} \sum_{k=1}^{n} w_{j} w_{k} S_{j}(0) S_{k}(0) \rho_{j, k} \sigma_{j} \sigma_{k}}}, \text { for } i=1,2, \ldots, n
$$

The prices $V^{S i m}, V^{c}$ and $V^{l}$ are calculated for strike prices $K_{i}=95,96, \ldots, 115$. The option prices are shown in Figure 1. Since a put option has a convex payoff, the price $V^{\text {Sim }}$ is bounded from above by $V^{c}$ and from below by $V^{l}$ for every $K_{i}$. We also observe that $V^{l}$ is a good approximation for the basket derivative price: $V^{l} \approx V^{S i m}$, whereas the upper bound does not succeed in providing a close approximation ${ }^{2}$. The reason is that the price $V^{c}$ does not contain any information about the dependence structure and assumes that all pairwise correlations are equal to one. Therefore, the accuracy of the upper bound $V^{c}$ is improving when the stocks composing the basket are moving strong together. Note that the computation time to generate the basket put option prices $V^{S i m}$ is around 4 seconds, whereas the upper bounds $V^{c}$ and lower bounds $V^{l}$ can be determined in approximately 0.4 seconds.

Table 1: The four-basket put option

\begin{tabular}{rrrrr}
\hline stocks & 1 & 2 & 3 & 4 \\
\hline Vols & 0.25 & 0.3 & 0.45 & 0.8 \\
Spot prices & 100 & 100 & 100 & 100 \\
Weights & 0.25 & 0.25 & 0.25 & 0.25 \\
& & & & \\
Correlation & 0.3 & & & \\
Maturity & 1 & & & \\
Risk-free rate & 0.01 & & & \\
\hline
\end{tabular}

Consider the four-basket put option with parameters given in Table 1 and strike price $K=100$. Since the lower bound prices $V^{l}$ provide close approximations for the real basket option price, we continue using the lower bound for approximating the real basket option prices. The comonotonic finite difference method determines a matrix of option prices $V_{j}^{k+1}$ defined in (4.4), where the volatility $\sigma_{i}$ has to be replaced by $\nu_{i} \sigma_{i}$ since we work in the lower bound framework. The price $V_{j}^{k}$ is an approximation for the time- $t_{k}$ price of the basket put with basket spot price given by $\sum_{i=1}^{n} w_{i} S_{i}(0) \mathrm{e}^{\left(r-\frac{1}{2}\left(\nu_{i} \sigma_{i}\right)^{2}\right) t_{k}+\nu_{i} \sigma_{i} b_{j}}$ and time to maturity given by $T-t_{k}$. The delta of an option is its sensitivity w.r.t. the underlying basket spot price. We can write the delta of the basket derivative $V^{l}$ as follows: $\Delta=\frac{\partial V^{l}}{\partial S^{l}}$. Using the chain rule, these deltas can be approximated in the finite difference scheme as follows

$$
\Delta_{j}^{k}=\frac{\frac{V_{j+1}^{k}-V_{j-1}^{k}}{2 \delta B}}{\sum_{i=1}^{n} \nu_{i} \sigma_{i} w_{i}\left(S_{i}\right)_{j}^{k}}
$$

where

$$
\left(S_{i}\right)_{j}^{k}=S_{i}(0) \mathrm{e}^{\left(r-\frac{1}{2}\left(\nu_{i} \sigma_{i}\right)^{2}\right) t_{k}+\nu_{i} \sigma_{i} b_{j}}
$$

The left panel of Figure 2 shows the basket put option prices $V_{j}^{k+1}$ based on the comonotonic lower bound approximation (2.7). This surface is an approximation for the real price of a basket put option

\footnotetext{
${ }^{2}$ The accuracy of the lower bound when approximating prices for European-type basket derivatives using risk-neutral valuation is already studied in Deelstra et al. (2004), Deelstra and Hainaut (2014) and Linders (2013).
} 


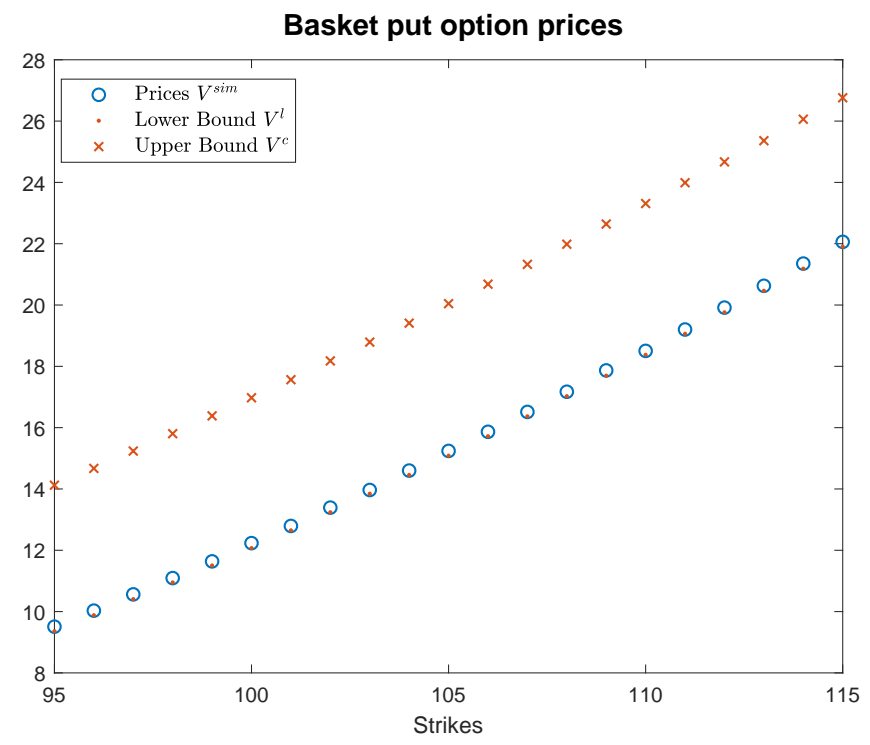

Figure 1: Basket put option prices $V_{K}^{\operatorname{Sim}}, V_{K}^{c}$ and $V_{K}^{l}$ for different strike values $K$ for the four-basket option with parameter values given in Table 1 .

for different basket spot prices and different times to maturity. The right panel of Figure 2 shows the corresponding deltas, based on a lower bound approximation. Note that calculating these price and delta surfaces does not require any extra work for the finite difference scheme.

\subsection{Comonotonic finite difference method for the lower bound of American-type basket options}

Consider an American-type basket derivative with payoff function $H$ and maturity $T$. We partition the time interval $[0, T]$ using a time step $\delta t$ and the space interval $[-I \delta B,(J-I) \delta B]$ using a step size $\delta B$. We take 15000 time steps per year, and the corresponding space grid is chosen such that it complies with the condition 4.5). We performed convergence tests on the finite different scheme which suggest that 15000 time steps is sufficient. We show now how the early exercise features can be implemented in the finite difference scheme, similarly to the one-dimensional Black \& Scholes case.

Assume we already derived the American-type basket derivative prices $V_{j}^{l}$, for $j=0,1, \ldots, J$ and $l=0,1, \ldots, k$. The American-type basket derivative price $V_{j}^{k+1}$ at the time point $t_{k+1}$ can be determined by comparing the present value of the contract in case the option is not exercised with the payoff of the derivative in case of early exercise at time $t_{k+1}$. In case the derivative is not exercised at time $t_{k+1}$, it behaves as a European-type derivative in the interval $\left[t_{k+1}, t_{k}\right]$. Therefore, the time- $t_{k+1}$ price in case of no early exercise is denoted by $\tilde{V}_{j}^{k+1}$ and can be determined by using the methodology explained in Section 4.1. We have that:

$$
\tilde{V}_{j}^{k+1}=\frac{1}{2} \frac{\delta t}{\delta B^{2}} V_{j-1}^{k}+\left(1-r \delta t-\frac{\delta t}{\delta B^{2}}\right) V_{j}^{k}+\frac{1}{2} \frac{\delta t}{\delta B^{2}} V_{j+1}^{k}+\mathcal{O}\left(\delta t ; \delta B^{2}\right),
$$

for $j=1, \ldots, J-1$ and $k=0,1, \ldots, N-1$. Note that we use a marginal volatility parameter equal to $\nu_{i} \sigma_{i}$ because we want to use the lower bound approximation as a close approximation for the real Americantype basket put option price. The holder of the derivative will only use the right to exercise at time $t_{k+1}$ 

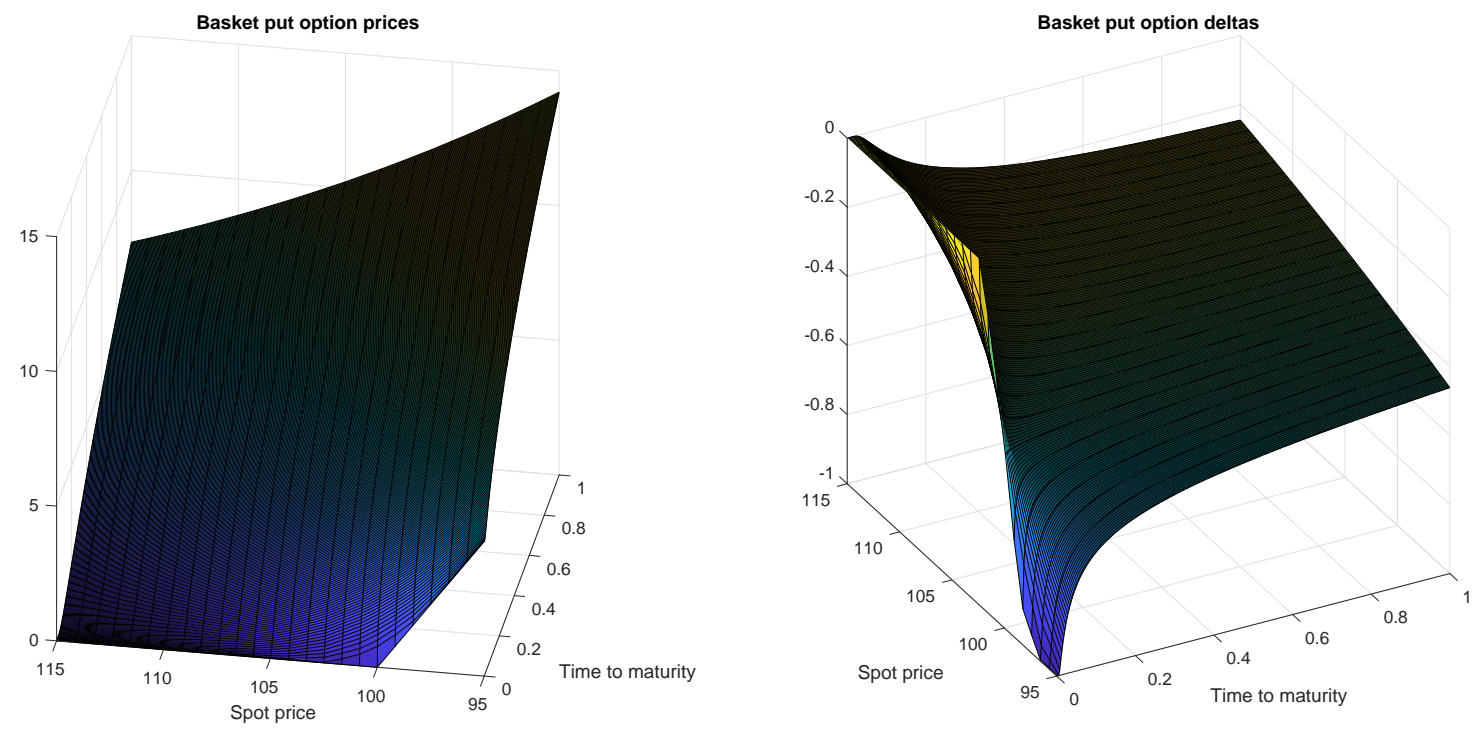

Figure 2: The left panel shows the option prices $V_{j}^{k+1}$ based on the lower bound approximation for the four-basket put option of Table 1 for different maturities and different spot prices. The right panel shows the corresponding deltas.

if the option provides a payoff which is larger than $\tilde{V}_{j}^{k+1}$. Therefore, the value $V_{j}^{k+1}$ corresponds with

$$
V_{j}^{k+1}=\max \left\{\tilde{V}_{j}^{k+1},\left(K-S_{j}^{k+1}\right)_{+}\right\}
$$

where

$$
S_{j}^{k+1}=\sum_{i=1}^{n} w_{i} S_{i}(0) \mathrm{e}^{\left(r-\frac{1}{2}\left(\nu_{i} \sigma_{i}\right)^{2}\right) t_{k+1}+\nu_{i} \sigma_{j} b_{j}}
$$

Additionally, the comonotonic finite difference method with the lower bound approximation allows to determine at each time point $t_{k}$ the basket price $S^{*}\left(t_{k}\right)$ such that it is optimal to exercise the basket put if the basket spot price is below $S^{*}\left(t_{k}\right)$. The function $S^{*}$ is called the exercise region and this exercise region can directly be extracted from the finite difference scheme without any extra computational cost. An approximate exercise region can be determined by using the comonotonic finite difference method together with the lower bound approximation. The result is shown in Figure 3

\section{Pricing American basket put options: comparison with the Least-Squares Monte Carlo}

In this section, we use the finite difference scheme to price American put options, and we compare the results with the Least-Squares method. We have already illustrated the accuracy of the proposed method in determining the upper bound $V^{c}$ and the lower bound $V^{l}$. Now we combine these bounds to determine an approximate price. First, consider a European-type basket put option with strike $K$ and maturity $T$. An 


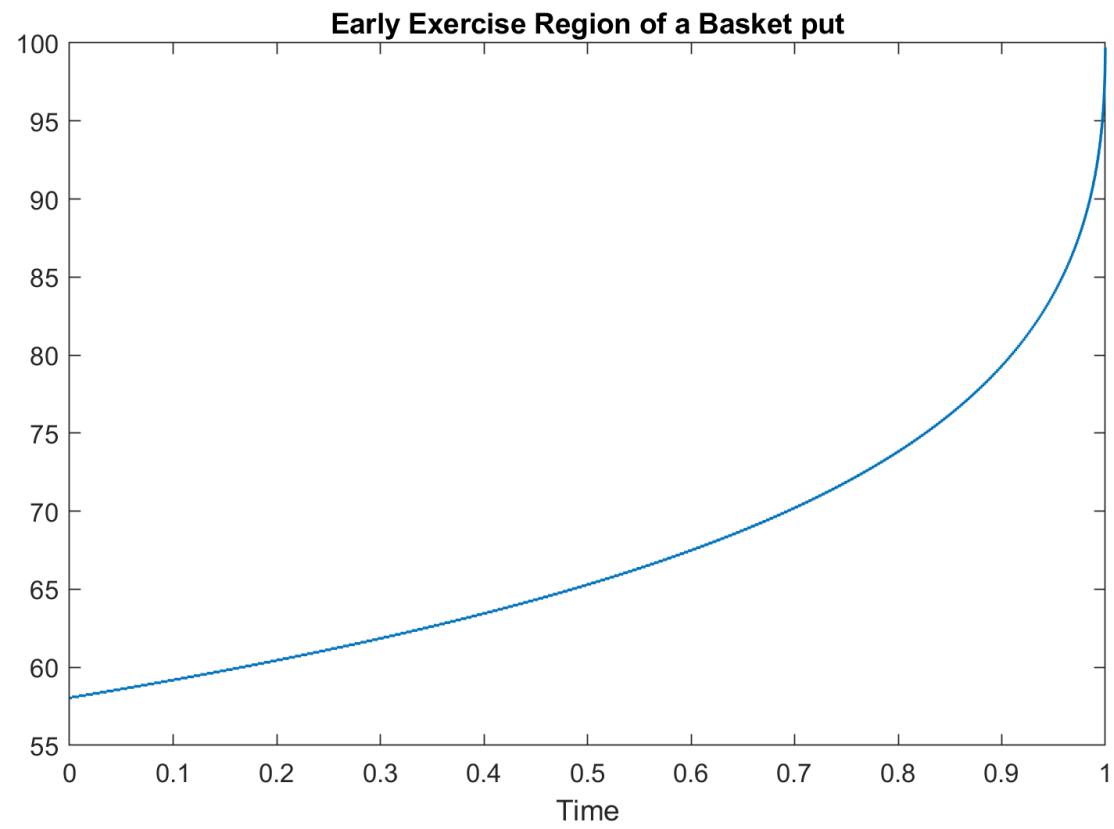

Figure 3: Exercise region for the lower bound approximation of the four-basket American-type put option with parameters given in Table 1 .

approximation for the real price $V\left(t, S_{1}, S_{2}, \ldots, S_{n} ; K\right)$ is denoted $\bar{V}\left(t, S_{1}, S_{2}, \ldots, S_{n} ; K\right)$ and follows from the linear combination:

$$
\bar{V}\left(t, S_{1}, S_{2}, \ldots, S_{n} ; K\right)=z V^{l}\left(t, S^{l} ; K\right)+(1-z) V^{c}\left(t, S^{c} ; K\right),
$$

and we aim to find a close approximation $\bar{V}$ for the real price $V$. It can be shown, see e.g. Vyncke et al. (2004) and Linders (2013), that by taking the weight $z$ such that:

$$
z=\frac{\operatorname{Var}\left[S^{c}(T) \mid \mathcal{F}_{t}\right]-\operatorname{Var}\left[S(T) \mid \mathcal{F}_{t}\right]}{\operatorname{Var}\left[S^{c}(T) \mid \mathcal{F}_{t}\right]-\operatorname{Var}\left[S^{l}(T) \mid \mathcal{F}_{t}\right]}
$$

the following relation holds:

$$
\int_{0}^{+\infty} \bar{V} \mathrm{~d} K=\int_{0}^{+\infty} V \mathrm{~d} K
$$

The variances needed to calculate $z$ are taken under the risk-neutral measure, and are given by:

$$
\begin{aligned}
\operatorname{Var}\left[S(T) \mid \mathcal{F}_{t}\right] & =\sum_{i=1}^{n} \sum_{j=1}^{n} w_{i} w_{j} S_{i}(t) S_{j}(t) \mathrm{e}^{2 r(T-t)}\left(\mathrm{e}^{\rho_{i, j} \sigma_{i} \sigma_{j}(T-t)}-1\right), \\
\operatorname{Var}\left[S^{c}(T) \mid \mathcal{F}_{t}\right] & =\sum_{i=1}^{n} \sum_{j=1}^{n} w_{i} w_{j} S_{i}(t) S_{j}(t) \mathrm{e}^{2 r(T-t)}\left(\mathrm{e}^{\sigma_{i} \sigma_{j}(T-t)}-1\right), \\
\operatorname{Var}\left[S^{l}(T) \mid \mathcal{F}_{t}\right] & =\sum_{i=1}^{n} \sum_{j=1}^{n} w_{i} S_{i}(t) w_{j} S_{j}(t) \mathrm{e}^{2 r(T-t)}\left(\mathrm{e}^{\nu_{i} \nu_{j} \sigma_{i} \sigma_{j}(T-t)}-1\right) .
\end{aligned}
$$

Therefore, relation (5.1) implies that an approximation for the basket option price can be obtained with two finite difference schemes for $V^{l}$ and $V^{c}$. The finite difference scheme is implemented as 
described in the previous section. For the LSM, we use 50 exercising times per year. The regressions are performed using standard polynomials up to the second degree for all stocks composing the basket. In particular, for the in-the-money paths at each time step, the payoff is approximated by the following linear model:

$$
\beta_{0}+\sum_{i=1}^{n} \beta_{1, i} \frac{S_{i}(t)}{S_{i}(0)}+\sum_{i=1}^{n} \beta_{2, i}\left(\frac{S_{i}(t)}{S_{i}(0)}\right)^{2} .
$$

The number of simulated paths is 100000 . Moreover, the displayed LSM prices are taken as the average among 10 simulated prices, which seems to be enough to reduce the variance due to the simulations, and not too much in order to keep the computation time reasonable. All calculations are performed using $\mathbf{R}$ software on a Dell computer with processor reference 'Intel(R) Core(TM) i7-4910MQ CPU 2.90GHz'. We rely on the mvrnorm() function in the MASS package to generate multivariate normal random variables (see Venables and Ripley (2002)) and the $\operatorname{lm}($ ) function for the linear regressions (see R Core Team (2017)). In order to simplify the numerical testing and without any loss of generality, we assume in the following illustrations that all pairs of stocks have the same correlation and we use the notation $\rho=\rho_{i j}$ for all $i \neq j$. Furthermore, we assume a constant interest rate of $1 \%$.

Table 2 displays American-type basket put option prices for an equally weighted basket of 8 stocks, all having the same initial value 40 . The volatilities of stocks 2 to 8 are $60 \%, 10 \%, 90 \%, 30 \%, 70 \%$, $80 \%$ and 20\%, respectively. Prices are calculated for 3 different maturities (i.e. 6 months, 1 year and 2 years), 3 different strikes (i.e. 35,40 and 45), two levels of the volatility of the first stock (i.e. $30 \%$ and $90 \%$ ) and two levels of the pairwise correlation (i.e. 0.3 and 0.8). Column 'Rel. diff.' reports the relative difference in percent between the price using the finite difference method and average price obtained with the LSM method. We observe that the two methods give very close prices. However, we see from columns 'Comp. time FD' and 'Comp. time LSM' that the computation time is significantly smaller for the finite different method. The first reason is that for longer maturities, the increase of both the number of simulations and the number of exercising opportunities slows down the process. Another factor impacting the computation time is the strike price. For higher values of the exercising price, the number of in-the-money paths is higher, requiring further computational efforts. We found the same order of relative differences and computation times in other examples where we take different weights in the basket, different basket sizes and different volatilities. The results are not reported here but are available to the reader upon request.

Figure 4 depicts sensitivity tests with respect to the maturity, the strike, the volatility of the first stock and the pairwise correlation. We focus on the relative differences between American-type basket put prices obtained with the finite difference method against those obtained with the LSM method. The basket under consideration is an equally weighted sum of 4 stocks with initial prices of 40 . The base case from which the sensitivities are drawn is such that the maturity is 1 year and the exercise price is 40 . The pairwise correlation is 0.3 and the volatilities of the four stocks are $\sigma_{1}=40 \%, \sigma_{2}=60 \%, \sigma_{3}=10 \%$ and $\sigma_{4}=90 \%$. The top-left graph shows the sensitivity with respect to the maturity, where we see that the differences are relatively small. However, it is worth to mention that for high maturities, the relative differences tend to increase. This can be due to the fact that the approximation errors in the LSM are compounded over the exercising times, which leads to biased prices. The top-right graph corresponds to the sensitivity with respect to the strike. It appears that the relative differences become too big for low strikes. One reason could be that for low strikes, the number of in-the-money paths in the LSM is reduced, such that the regressions are not performing very well. The same reason could explain the increase of the relative difference for high volatilities in the bottom left graph. Finally, the bottom-right graph displays the relative differences as a function of the pairwise correlation coefficient where we see that the differences are often very small. 
Table 2: American-type basket put option prices on a basket of 8 equally weighted stocks with initial prices 40 computed using finite difference (FD) method and the LSM with the relative differences (in \%) and the corresponding computation times (in seconds).

\begin{tabular}{|c|c|c|c|c|c|c|c|c|}
\hline Maturity & Strike & $\sigma_{1}$ & $\rho$ & FD prices & LSM prices & Rel. diff. & Comp. time FD & Comp. time LSM \\
\hline \multirow[t]{12}{*}{6 months } & \multirow[t]{4}{*}{35} & \multirow[t]{2}{*}{0.3} & 0.3 & 1.279 & 1.282 & 0.219 & 9.36 & 79.81 \\
\hline & & & 0.8 & 2.408 & 2.413 & 0.228 & 9.17 & 96.44 \\
\hline & & \multirow[t]{2}{*}{0.9} & 0.3 & 1.700 & 1.703 & 0.181 & 9.17 & 86.71 \\
\hline & & & 0.8 & 3.053 & 3.064 & 0.369 & 9.17 & 108.04 \\
\hline & \multirow[t]{4}{*}{40} & \multirow[t]{2}{*}{0.3} & 0.3 & 3.461 & 3.468 & 0.183 & 9.36 & 164.88 \\
\hline & & & 0.8 & 4.866 & 4.879 & 0.256 & 10.33 & 175.81 \\
\hline & & \multirow[t]{2}{*}{0.9} & 0.3 & 3.989 & 4.008 & 0.453 & 10.39 & 163.91 \\
\hline & & & 0.8 & 5.618 & 5.620 & 0.023 & 9.57 & 157.50 \\
\hline & \multirow[t]{4}{*}{45} & \multirow[t]{2}{*}{0.3} & 0.3 & 6.742 & 6.739 & 0.037 & 9.33 & 216.22 \\
\hline & & & 0.8 & 8.109 & 8.118 & 0.104 & 9.49 & 201.32 \\
\hline & & \multirow[t]{2}{*}{0.9} & 0.3 & 7.236 & 7.245 & 0.132 & 9.33 & 203.58 \\
\hline & & & 0.8 & 8.860 & 8.881 & 0.232 & 8.98 & 196.07 \\
\hline \multirow{12}{*}{1 year } & \multirow{4}{*}{35} & \multirow{2}{*}{0.3} & 0.3 & 2.386 & 2.374 & 0.486 & 18.78 & 231.91 \\
\hline & & & 0.8 & 4.015 & 4.019 & 0.100 & 18.34 & 270.99 \\
\hline & & \multirow[t]{2}{*}{0.9} & 0.3 & 3.033 & 3.032 & 0.030 & 18.09 & 246.30 \\
\hline & & & 0.8 & 4.945 & 4.946 & 0.022 & 18.22 & 280.50 \\
\hline & \multirow[t]{4}{*}{40} & \multirow[t]{2}{*}{0.3} & 0.3 & 4.848 & 4.837 & 0.237 & 17.97 & 1601.22 \\
\hline & & & 0.8 & 6.743 & 6.732 & 0.159 & 84.94 & 1557.22 \\
\hline & & \multirow[t]{2}{*}{0.9} & 0.3 & 5.596 & 5.596 & 0.010 & 85.59 & 1515.75 \\
\hline & & & 0.8 & 7.784 & 7.787 & 0.035 & 81.05 & 1541.76 \\
\hline & \multirow[t]{4}{*}{45} & \multirow{2}{*}{0.3} & 0.3 & 8.093 & 8.079 & 0.169 & 84.80 & 1977.86 \\
\hline & & & 0.8 & 10.027 & 10.016 & 0.109 & 82.61 & 1281.59 \\
\hline & & \multirow[t]{2}{*}{0.9} & 0.3 & 8.093 & 8.842 & 0.077 & 18.24 & 474.82 \\
\hline & & & 0.8 & 11.098 & 11.085 & 0.120 & 17.85 & 455.61 \\
\hline \multirow[t]{12}{*}{2 years } & \multirow[t]{4}{*}{35} & \multirow[t]{2}{*}{0.3} & 0.3 & 4.035 & 3.974 & 1.521 & 37.36 & 729.34 \\
\hline & & & 0.8 & 6.196 & 6.183 & 0.216 & 37.30 & 2374.89 \\
\hline & & \multirow[t]{2}{*}{0.9} & 0.3 & 4.978 & 4.954 & 0.492 & 167.00 & 3028.90 \\
\hline & & & 0.8 & 7.481 & 7.477 & 0.065 & 163.18 & 3454.50 \\
\hline & \multirow[t]{4}{*}{40} & \multirow[t]{2}{*}{0.3} & 0.3 & 6.775 & 6.704 & 1.041 & 169.78 & 4023.14 \\
\hline & & & 0.8 & 9.204 & 9.176 & 0.299 & 165.61 & 4148.22 \\
\hline & & 0.9 & 0.3 & 7.822 & 7.814 & 0.101 & 167.06 & 4055.97 \\
\hline & & & 0.8 & 10.614 & 10.594 & 0.187 & 163.75 & 4245.87 \\
\hline & 45 & 0.3 & 0.3 & 10.065 & 10.011 & 0.538 & 170.83 & 4990.59 \\
\hline & & & 0.8 & 12.602 & 12.576 & 0.209 & 165.97 & 4862.00 \\
\hline & & 0.9 & 0.3 & 11.136 & 11.130 & 0.049 & 167.44 & 4911.06 \\
\hline & & & 0.8 & 14.075 & 14.049 & 0.188 & 164.21 & 4836.68 \\
\hline
\end{tabular}



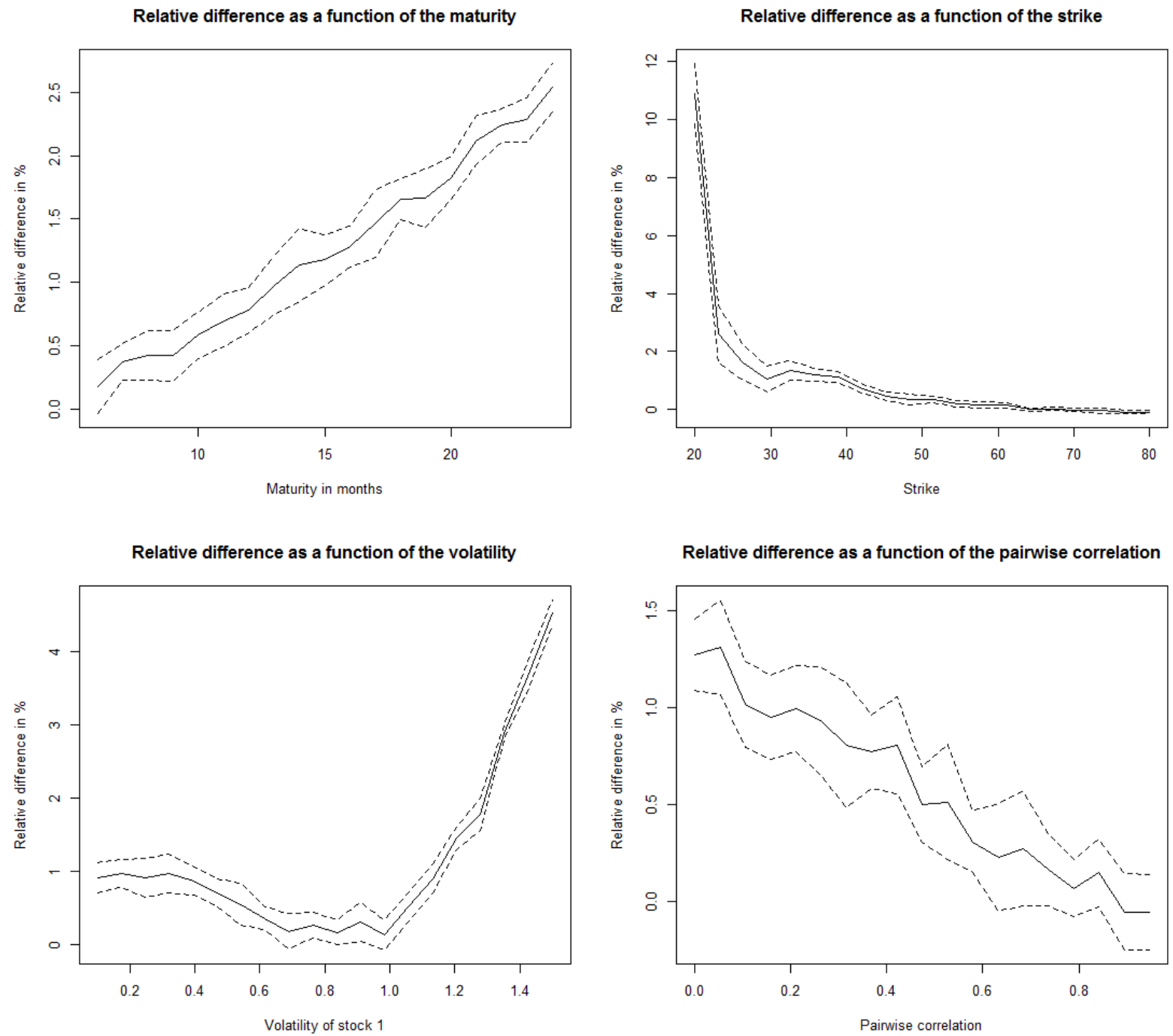

Figure 4: Relative difference between the finite difference method and the LSM as a function of the maturity (top-left), strike (top-right), volatility of stock 1 (bottom-left) and pairwise correlation (bottomright) for an American-type basket put option, where the basket is composed of 4 equally weighted stocks with initial prices 40 . The dashed lines are the differences with upper and lower bounds of the $95 \%$ confidence interval 
Table 3: American-type basket put option prices on a basket of 4 equally weighted stocks with initial prices 40 for high pairwise correlations.

\begin{tabular}{cccc}
\hline Pairwise correlation & FD prices & LSM prices & Comonotonic LSM \\
\hline 0.95 & 7.427 & 7.433 & - \\
0.96 & 7.452 & 7.444 & - \\
0.97 & 7.476 & 7.476 & - \\
0.98 & 7.500 & 7.511 & - \\
0.99 & 7.524 & 8.670 & - \\
0.992 & 7.529 & 10.250 & - \\
0.994 & 7.534 & 10.234 & - \\
0.996 & 7.538 & 14.702 & - \\
0.998 & 7.543 & 17.212 & - \\
1.000 & 7.548 & 259.274 & 7.550 \\
\hline
\end{tabular}

However, surprising values are observed when the correlation coefficient approaches 1 , which are reported in Table 3 but not in Figure 4 . While the finite difference method returns smooth prices (i.e. from 7.43 to 7.55 for correlation coefficients between 0.95 and 1), the LSM prices increase very fast from 7.43 for $\rho=0.95$ to 17.2 for $\rho=0.998$, and up to 259.3 in the comonotonic case; see Table 3 . The reason for this deficiency of the LSM could be that the interpolation becomes stiff for high correlations 3 In order to better understand how the LSM performs in the comonotonic case, we look at the values of the regression coefficients and their significance level at a given exercising time. Table 4 displays the estimates of the regression coefficients and their corresponding $p$-values for pairwise correlations $0.5,0.95$ and 1 at the exercising time 25. We observe that for a correlation coefficient equal to 0.5 , all $p$-values are very close to 0 , indicating that all components of the linear regression are highly significant. For a pairwise correlation of 0.95 , two regressors are not significant anymore, but the estimates of their parameters remain close to those obtained for a correlation equal to 0.5 . In the comonotonic case, none of the regressors is significant and their estimates are either too high or too low compared to those obtained for $\rho=0.5$ and $\rho=0.95$. Moreover, the algorithm fails to provide an estimate for $\beta_{2,3}$. The reason for all these anomalies could be that in the comonotonic case, knowing the realization of one component is sufficient to determine the realization of the whole basket, provided the marginal distributions are known. Thus, including all basket components might lead to overfitting. Therefore, we propose to enhance the LSM method in the comonotonic case by including in the regressions only one of the assets composing the basket, namely, the first asset. This adjustment significantly improves the method. On the one hand, we observe in Table 5 that all coefficients are significant, and on the other hand, the adjusted algorithm leads to a price of 7.55 in the comonotonic case, which is in line with the corresponding FD price.

Next, we compare the efficiency of the two methods in terms of computation time when the basket size increases. As we have seen earlier, the proposed partial differential equation does not depend on the number of components composing the basket. However, the implementation of the finite difference scheme requires a grid for the shifted Brownian motion, which is then plugged into each stock in order to obtain a partitioning of the payoff. For baskets with bigger size, this procedure can slow down the algorithm, but the overall computation cost remains affordable. For the LSM, it is necessary to simulate the paths of driving Brownian motions for each stock, which results in a steep increase in computation time. Furthermore, although the least-squares estimation contributes only marginally in the overall computation time, it also slows down the algorithm when the basket size increases. Figure 5 displays the

\footnotetext{
${ }^{3}$ We thank the referee for pointing out the need of a more detailed investigation.
} 
Table 4: Coefficients and corresponding $p$-values (in brackets) from the linear regression of the LSM at the exercising time 25 for pairwise correlations $0.5,0.95$ and 1 .

\begin{tabular}{cccc}
\hline Coefficients & $\rho=0.5$ & $\rho=0.95$ & $\rho=1$ \\
\hline$\beta_{0}$ & 56.36 & 47.85 & 9383.1 \\
& $\left(<2 \times 10^{-16}\right)$ & $\left(4 \times 10^{-05}\right)$ & $(0.508)$ \\
$\beta_{1,1}$ & -12.17 & -14.86 & 101543.8 \\
& $\left(<2 \times 10^{-16}\right)$ & $\left(4 \times 10^{-09}\right)$ & $(0.521)$ \\
$\beta_{1,2}$ & -11.43 & -12.82 & -268656.9 \\
& $\left(<2 \times 10^{-16}\right)$ & $\left(<2 \times 10^{-16}\right)$ & $(0.530)$ \\
$\beta_{1,3}$ & -46.10 & -22.96 & -24649.0 \\
& $\left(7 \times 10^{-09}\right)$ & $(0.369)$ & $(0.511)$ \\
$\beta_{1,4}$ & -9.83 & -13.78 & -351436.8 \\
& $\left(<2 \times 10^{-16}\right)$ & $\left(<2 \times 10^{-16}\right)$ & $(0.547)$ \\
$\beta_{2,1}$ & 2.44 & 4.79 & 460461.3 \\
& $\left(4 \times 10^{-10}\right)$ & $(0.0009)$ & $(0.541)$ \\
$\beta_{2,2}$ & 2.42 & 3.90 & 28192.3 \\
& $\left(<2 \times 10^{-16}\right)$ & $\left(2 \times 10^{-05}\right)$ & $(0.569)$ \\
$\beta_{2,3}$ & 19.54 & 7.44 & Not available \\
& $\left(1.5 \times 10^{-06}\right)$ & $(0.575)$ & \\
$\beta_{2,4}$ & 2.27 & 7.74 & -909.1 \\
& $\left(<2 \times 10^{-16}\right)$ & $\left(<2 \times 10^{-16}\right)$ & $(0.624)$ \\
\hline
\end{tabular}

Table 5: Coefficients and corresponding $p$-values (in brackets) from the linear regression of the adjusted comonotonic LSM at the exercising time 25.

\begin{tabular}{cc}
\hline Coefficients & $\rho=1$ \\
\hline$\beta_{0}$ & 42.54 \\
& $\left(<2 \times 10^{-16}\right)$ \\
$\beta_{1,1}$ & -48.52 \\
& $\left(<2 \times 10^{-16}\right)$ \\
$\beta_{2,1}$ & 11.78 \\
& $\left(<2 \times 10^{-16}\right)$ \\
\hline
\end{tabular}




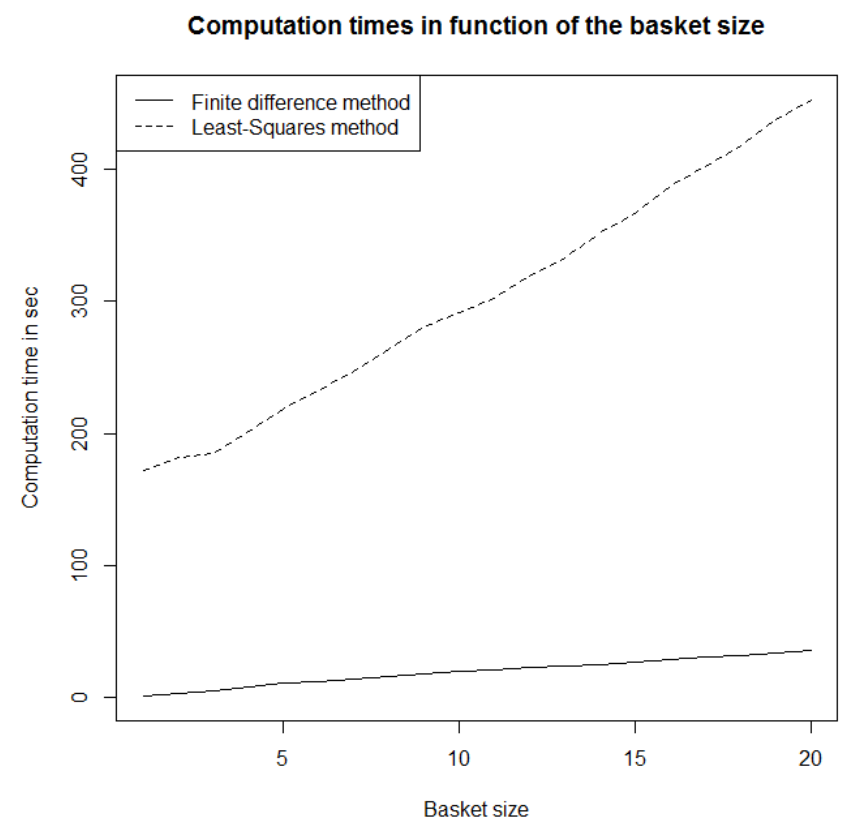

Figure 5: Computation times of the LSM and the FDM as a function of the basket size with 1 year maturity and strike 40 . All components have a spot price of 40 and a volatility of $30 \%$, and the pairwise correlation is 0.3 .

computation time for both methods, and we clearly see a significant gain in computation time for the finite difference method (up to 30 times faster for small basket sizes and 10 times faster for bigger baskets). Therefore, given its accuracy and speed, the proposed finite difference scheme seems to be much more efficient than the standard Least-Squares method.

\section{Concluding remarks}

We propose a simple PDE method to price American-type basket derivatives using the theory of comonotonicity. We reduce the initial multi-dimensional problem to a univariate problem. The obtained comonotonic PDE does not depend on the number of stocks in the basket and can be solved to find an explicit expression for the basket derivative price. American-type basket derivative prices can be determined by numerically solving the comonotonic PDE with a finite difference method. Our results on the comonotonic market are used to determine basket derivative prices in a non-comonotonic market with correlated stocks. We show that American-type basket derivative prices determined using our methodology are close to the corresponding prices determined using the LSM method. However, we also show that our methodology outperforms the LSM method in a number of significant aspects, such as the computation time and the derivation of the exercise region.

\section{References}

Ahmad, R. (2006), Numerical and computational methods for derivative pricing, John Wiley \& Sons.

Björk, T. (1998), Arbitrage theory in continuous time, Oxford University Press. pp. 311. 
Brennan, M. J. and Schwartz, E. S. (1977), 'The valuation of American put options', Journal of Finance 32, 449-462. https://doi.org/10.1111/j.1540-6261.1977.tb03284.x.

Caldana, R., Fusai, G., Gnoatto, A. and Graselli, M. (2016), 'General close-form basket option pricing bounds', Quantitative Finance 16, 535-554. https://doi.org/10.1080/14697688.2015.1073854.

Carslow, H. S. and Jaeger, J. C. (1959), Conduction of heat in solids, Clarendon Press, Oxford.

Chen, X., Deelstra, G., Dhaene, J., Linders, D. and Vanmaele, M. (2015), 'On an optimization problem related to static super-replicating strategies', Journal of Computational and Applied Mathematics 278, 213-230. https://doi.org/10.1016/j.cam.2014.10.003.

Chen, X., Deelstra, G., Dhaene, J. and Vanmaele, M. (2008), 'Static super-replicating strategies for a class of exotic options', Insurance: Mathematics \& Economics 42, 1067-1085. https://doi.org/10.1016/j.insmatheco.2008.01.002

Clift, S. S. and Forsyth, P. A. (2008), 'Numerical solution of two asset jump diffusion models for option valuation', Applied Numerical Mathematics 58, 743-782. https://doi.org/10.1016/j.apnum.2007.02.005.

Company, R., Egorova, V., Jódar, L. and Soleymani, F. (2016), 'A mixed derivative terms removing method in multi-asset option pricing problems', Applied Mathematics Letters 60, 108-114. https://doi.org/10.1016/j.aml.2016.04.011.

Crank, J. (1975), The mathematics of diffusion (2nd ed.), Oxford University Press.

Crank, J. and Nicolson, P. (1947), A practical method for numerical evaluation of solutions of partial differential equations of the heat conduction type, in 'Proceedings of the Cambridge Philosophical Society', Vol. 43, pp. 50-67.

Deelstra, G., Diallo, I. and Vanmaele, M. (2008), 'Bounds for Asian basket options', Journal of Computational and Applied Mathematics 218, 215-228. https://doi.org/10.1016/j.cam.2006.12.017.

Deelstra, G. and Hainaut, D. (2014), 'Valuation and default probabilities of a holding company based on comonotone approximations', Journal of Computational and Applied Mathematics .

Deelstra, G., Liinev, J. and Vanmaele, M. (2004), 'Pricing of arithmetic basket options by conditioning', Insurance: Mathematics and Economics 34, 55-77. https://doi.org/10.1016/j.insmatheco.2003.11.002.

Dhaene, J., Denuit, M., Goovaerts, M. J., Kaas, R. and Vyncke, D. (2002a), 'The concept of comonotonicity in actuarial science and finance: theory', Insurance: Mathematics and Economics 31, 3-33. https://doi.org/10.1016/S0167-6687(02)00134-8.

Dhaene, J., Denuit, M., Goovaerts, M. J., Kaas, R. and Vyncke, D. (2002b), 'The concept of comonotonicity in actuarial science and finance: applications', Insurance: Mathematics and Economics 31, 133-161. https://doi.org/10.1016/S0167-6687(02)00135-X.

Dhaene, J., Kukush, A. and Linders, D. (2014), 'The multivariate Black \& Scholes market: Conditions for completeness and no-arbitrage', Theory of Probability and Mathematical Statistics 88, 85-98. https://doi.org/10.1090/S0094-9000-2014-00920-5.

Dhaene, J., Kukush, A. and Linders, D. (2018), Comonotonic asset prices in arbitrage-free markets, Working Paper, Leuven: KU Leuven - Faculty of Business and Economics. 
Dhaene, J., Wang, S., Young, V. and Goovaerts, M. J. (2000), 'Comonotonicity and maximal stop-loss premiums', Bulletin of the Swiss Association of Actuaries 5, 99-113.

Duffy, D. (2004), 'A critique of the Crank-Nicolson scheme', Wilmott magazine July 2004, 68-77.

Düring, B. and Heuer, C. (2015), 'High-order compact schemes for parabolic problems with mixed derivatives in multiple space dimensions', SIAM Journal on Numerical Analysis 53, 2113-2134. https://doi.org/10.1137/140974833.

Escobar, M., Krause, D. and Zagst, R. (2016), 'Stochastic covariance and dimension reduction in the pricing of basket options', Review of Derivatives Research 19(3), 165-200.

Haentjens, T. and In't Hout, K. J. (2015), 'ADI schemes for pricing American options under the Heston model', Applied Mathematical Finance 22, 207-237. https://doi.org/10.1080/1350486X.2015.1009129.

Hill, J. M. and Dewynne, J. N. (1987), Heat Conduction, Blackwell Scientific Publications, Oxford [Oxfordshire]; Boston.

Kaas, R., Dhaene, J. and Goovaerts, M. J. (2000), 'Upper and lower bounds for sums of random variables', Insurance: Mathematics and Economics 27, 151-168. https://doi.org/10.1016/S01676687(00)00060-3.

Krekel, M., de Kock, J., Korn, R. and Man, T.-K. (2006), 'An analysis of pricing methods for baskets options', The Best of Wilmott 2, 181-188.

Leentvaar, C. C. W. and Oosterlee, C. W. (2008), 'Multi-asset option pricing using a parallel Fourier-based technique', Journal of Computational Finance 12, 1-26. https://doi.org/10.21314/JCF.2008.184.

Linders, D. (2013), Pricing index options in a multivariate Black \& Scholes model, Research report AFI-1383, FEB, KU Leuven.

Linders, D. and Stassen, B. (2016), 'The multivariate Variance Gamma model: basket option pricing and calibration', Quantitative Finance 16, 555-572. https://doi.org/10.1080/14697688.2015.1043934.

Longstaff, F. A. and Schwarts, E. S. (2001), 'Valuing American options by simulations: a simple LeastSquares approach', The review of Financial Studies 14, 113-147.

Musiela, M. and Rutkowski, M. (2005), Martingale methods in financial modelling (2nd ed.), SpringerVerlag, Berlin.

R Core Team (2017), R: A Language and Environment for Statistical Computing, R Foundation for Statistical Computing, Vienna, Austria.

URL: https://www.R-project.org/

Reisinger, C. and Wittum, G. (2007), 'Efficient hierarchical approximation of highdimensional option pricing problems', SIAM Journal on Scientific Computing 29, 440-458. https://doi.org/10.1137/060649616.

Simon, S., Goovaerts, M. J. and Dhaene, J. (2000), 'An easy computable upper bound for the price of an arithmetic Asian option', Insurance: Mathematics and Economics 26, 175-183. https://doi.org/10.1016/S0167-6687(99)00051-7. 
Venables, W. N. and Ripley, B. D. (2002), Modern Applied Statistics with S, fourth edn, Springer, New York. ISBN 0-387-95457-0.

URL: http://www.stats.ox.ac.uk/pub/MASS4

Vyncke, D., Goovaerts, M. and Dhaene, J. (2004), 'An accurate analytical approximation for the price of a European-style arithmetic Asian option', Finance 25, 121-139.

Wang, Y. and Caflisch, R. (2010), 'Pricing and heding american-style options: a simple simulation-based approach', The Journal of Computational Finance 13(4), 95-125.

Wilmott, P. (2006), Paul Wilmott on Quantitative Finance, John Wiley \& Sons.

Wilmott, P., Dewynne, J. and Howison, S. (1994), Option pricing: Mathematical models and computation, Oxford Financial Press.

Witelski, T. P. and Bowen, M. (2003), 'ADI schemes for higher-order nonlinear diffusion equations', Applied Numerical Mathematics 45, 331-351. https://doi.org/10.1016/S0168-9274(02)00194-0

Zvan, R., Forsyth, P. A. and Vetzal, K. (1998), 'Robust numerical methods for PDE models of Asian options', Journal of Computational Finance 1, 39-78. https://doi.org/10.21314/JCF.1997.006.

\section{A Proof of Theorem 3.1}

The portfolio value $\Pi$ of the trading strategy $\pi$ is given by:

$$
\Pi(t)=\pi_{1}(t) S^{c}(t)+\pi_{2}(t) D(t) .
$$

Since $\pi$ is a replicating strategy for the derivative price $V_{\lambda}^{c}$, we should have that:

$$
\Pi(t)=V_{\lambda}^{c}\left(t, B_{\lambda}\right)
$$

and it follows that

$$
\pi_{2}(t)=\frac{1}{D(t)}\left(V_{\lambda}^{c}\left(t, B_{\lambda}\right)-\pi_{1}(t) S^{c}(t)\right) .
$$

The portfolio $\pi$ has to be self-financing, hence we find:

$$
\mathrm{d} \Pi=r V^{c} \mathrm{~d} t+\pi_{1} \sigma^{c} \mathrm{~d} B_{\lambda} .
$$

where we used (A.3) and (3.4) to rewrite the second term on the r.h.s.

On the other hand, for $V_{\lambda}^{c}\left(t, B_{\lambda}\right)$ in $\mathcal{C}^{2}\left(\mathbb{R}_{+} \times \mathbb{R}, \mathbb{R}\right)$, we apply Ito's Lemma together with (3.5) to derive:

$$
\mathrm{d} V_{\lambda}^{c}=\left(\frac{\partial V_{\lambda}^{c}}{\partial t}+\frac{1}{2} \frac{\partial^{2} V_{\lambda}^{c}}{\partial B_{\lambda}^{2}}\right) \mathrm{d} t+\frac{\partial V_{\lambda}^{c}}{\partial B_{\lambda}} \mathrm{d} B_{\lambda} .
$$

We define the stochastic process $Z=\{Z(t) \mid t \geq 0\}$ as follows:

$$
Z(t)=V^{c}(t)-\Pi(t) .
$$

Then it follows from (A.4) and (A.5) that:

$$
\mathrm{d} Z=\left(\frac{\partial V_{\lambda}^{c}}{\partial t}+\frac{1}{2} \frac{\partial^{2} V_{\lambda}^{c}}{\partial B_{\lambda}^{2}}-r V_{\lambda}^{c}\right) \mathrm{d} t+\left(\frac{\partial V_{\lambda}^{c}}{\partial B_{\lambda}}-\pi_{1} \sigma^{c}\right) \mathrm{d} B_{\lambda},
$$


and using [3.5), we find:

$$
\mathrm{d} Z=\left(\frac{\partial V_{\lambda}^{c}}{\partial t}+\frac{1}{2} \frac{\partial^{2} V_{\lambda}^{c}}{\partial B_{\lambda}^{2}}-r V_{\lambda}^{c}+\lambda\left(\frac{\partial V_{\lambda}^{c}}{\partial B_{\lambda}}-\pi_{1} \sigma^{c}\right)\right) \mathrm{d} t+\left(\frac{\partial V_{\lambda}^{c}}{\partial B_{\lambda}}-\pi_{1} \sigma^{c}\right) \mathrm{d} B
$$

Since A.2 holds, we should have that $\mathrm{d} Z=0$. Then, the uniqueness of the canonical decomposition for continuous semi-martingales leads to the conclusion that for any $t \in[0, T]$, the following integral

$$
\int_{0}^{t}\left(\frac{\partial V_{\lambda}^{c}}{\partial B_{\lambda}}(u)-\pi_{1}(u) \sigma^{c}(u)\right) \mathrm{d} B(u)=0,
$$

is $\mathbb{P}$-a.s. equal to zero. Since A.7 is an Ito integral, we can write:

$$
\int_{0}^{T}\left(\frac{\partial V_{\lambda}^{c}}{\partial B_{\lambda}}(u)-\pi_{1}(u) \sigma^{c}(u)\right)^{2} \mathrm{~d} u=0
$$

from which we find:

$$
\pi_{1}=\frac{1}{\sigma^{c}} \frac{\partial V_{\lambda}^{c}}{\partial B_{\lambda}}
$$

Using this choice for $\pi_{1}$ in the SDE (A.6), we find that

$$
\mathrm{d} Z=\left(\frac{\partial V_{\lambda}^{c}}{\partial t}+\frac{1}{2} \frac{\partial^{2} V_{\lambda}^{c}}{\partial B_{\lambda}^{2}}-r V_{\lambda}^{c}\right) \mathrm{d} t
$$

Hence, in order for $\mathrm{d} Z=0$ to hold, we should additionally have that the drift term is zero, which means that the following partial differential equation should be satisfied:

$$
\frac{\partial V_{\lambda}^{c}}{\partial t}+\frac{1}{2} \frac{\partial^{2} V_{\lambda}^{c}}{\partial B_{\lambda}^{2}}-r V_{\lambda}^{c}=0
$$

We conclude this proof by showing that the trading strategy $\pi$ is a self-financing strategy, provided (3.8) holds. We use A.5 and equality $\Pi(t)=V_{\lambda}^{c}\left(t, B_{\lambda}\right)$ to find:

$$
\mathrm{d} \Pi=\frac{\partial V_{\lambda}^{c}}{\partial B_{\lambda}} \mathrm{d} B_{\lambda}+\left(\frac{\partial V_{\lambda}^{c}}{\partial t}+\frac{1}{2} \frac{\partial^{2} V_{\lambda}^{c}}{\partial B_{\lambda}^{2}}\right) \mathrm{d} t .
$$

Taking into account $(3.8)$, we find:

$$
\mathrm{d} \Pi=\frac{\partial V_{\lambda}^{c}}{\partial B_{\lambda}} \mathrm{d} B_{\lambda}+r V_{\lambda}^{c} \mathrm{~d} t
$$

If we now use the SDE (3.6) for the process $S^{c}$, the SDE of $\Pi$ becomes:

$$
\mathrm{d} \Pi=\pi_{1} \mathrm{~d} S^{c}+\pi_{2} \mathrm{~d} D,
$$

which shows that $\pi$ is self-financing, and hence, concludes the proof.

\section{B Proof of Theorem 3.2}

We introduce the half time decay parameter $\tau=\frac{1}{2}(T-t)$ and the undiscounted price $U$, such that:

$$
V_{\lambda}^{c}\left(t, B_{\lambda}\right)=\mathrm{e}^{-2 r \tau} U\left(\tau, B_{\lambda}\right)
$$


The partial derivatives of $V$ can then be expressed as follows:

$$
\begin{aligned}
\frac{\partial V_{\lambda}^{c}}{\partial t} & =r \mathrm{e}^{-2 r \tau} U-\frac{1}{2} \mathrm{e}^{-2 r \tau} \frac{\partial U}{\partial \tau} \\
\frac{\partial V_{\lambda}^{c}}{\partial B_{\lambda}} & =\mathrm{e}^{-2 r \tau} \frac{\partial U}{\partial B_{\lambda}} \\
\frac{\partial^{2} V_{\lambda}^{c}}{\partial B_{\lambda}^{2}} & =\mathrm{e}^{-2 r \tau} \frac{\partial^{2} U}{\partial B_{\lambda}^{2}} .
\end{aligned}
$$

If we substitute these partial derivatives in the comonotonic PDE (3.8) and we use the change of variable $B_{\lambda}=x$, we find the following differential equation for $U$ :

$$
\frac{\partial U}{\partial \tau}=\frac{\partial^{2} U}{\partial x^{2}}
$$

Equation (B.1) is the so-called heat equation and can be solved using similarity reduction. More details on the heat equation with discussion on the similarity reduction can be found in Hill and Dewynne (1987). The fundamental solution $U_{f}$ satisfying the heat equation $\mathrm{B} .1$ is given by:

$$
U_{f}(\tau, x)=\frac{1}{2 \sqrt{\pi \tau}} \mathrm{e}^{-\frac{x^{2}}{4 \tau}}
$$

which corresponds with the pdf of a normal distribution with mean 0 and volatility $2 \tau$; see e.g. Carslow and Jaeger (1959) and Crank (1975) for a detailed discussion on solving the heat equation.

The general solution $U$, which is now satisfying the final condition

$$
U(0, x)=H\left(\sum_{i=1}^{n} w_{i} S_{i}(0) \mathrm{e}^{2\left(r-\frac{1}{2} \sigma_{i}^{2}\right) T+\sigma_{i} x}\right) .
$$

is then given by

$$
U(\tau, x)=\int_{-\infty}^{+\infty} \frac{1}{2 \sqrt{\pi \tau}} \mathrm{e}^{-\frac{(x-z)^{2}}{4 \tau}} U(0, x-z) \mathrm{d} z .
$$

Using the substitution $z=y+x$ and changing the coordinate system back to $\left(t, B_{\lambda}\right)$, the solution $V_{\lambda}^{c}$ can be written as:

$$
V_{\lambda}^{c}\left(t, B_{\lambda}\right)=\mathrm{e}^{-r(T-t)} \int_{-\infty}^{+\infty} \frac{\mathrm{e}^{-\frac{y^{2}}{2(T-t)}}}{\sqrt{2 \pi(T-t)}} H\left(\sum_{i=1}^{n} w_{i} S_{i}(0) \mathrm{e}^{\left(r-\frac{1}{2} \sigma_{i}^{2}\right) T+\sigma_{i}\left(B_{\lambda}+y\right)}\right) \mathrm{d} y .
$$

Given the realization $B_{\lambda}$ of the shifted Brownian motion at time $t$, the comonotonic stock prices $S_{i}^{c}(t)$ can be determined using (3.12) and we find (3.11).

\section{Proof of Theorem 4.1}

Let $V_{j}^{k}$ be the approximate derivative price obtained by employing the finite difference method (4.4). We take $\hat{V}_{j}^{k}$ as the corresponding approximation such that

$$
\hat{V}_{j}^{k}=V_{j}^{k}+\varepsilon_{j}^{k}
$$


where $\varepsilon_{j}^{k}$ is the error term at the node $(j, k)$, which satisfies 4.4, , since this equation also applies to $\hat{V}_{j}^{k}$ and $V_{j}^{k}$. Furthermore, we assume that the error is oscillatory of amplitude $\nu$ and frequency $\omega$, i.e.

$$
\varepsilon_{j}^{k}=\nu^{k} \exp (\mathrm{i} j \omega)
$$

where $\mathrm{i}^{2}=-1$. This leads to the following scheme for the error term:

$$
\nu^{k+1} \exp (\mathrm{i} j \omega)=\frac{1}{2} \frac{\delta t}{\delta B^{2}} \nu^{k}(\exp (\mathrm{i} j \omega-\mathrm{i} \omega)+\exp (\mathrm{i} j \omega+\mathrm{i} \omega))+\left(1-r \delta t-\frac{\delta t}{\delta B^{2}}\right) \nu^{k} \exp (\mathrm{i} j \omega),
$$

or, equivalently

$$
\nu=\frac{\delta t}{\delta B^{2}} \cos \omega+\left(1-r \delta t-\frac{\delta t}{\delta B^{2}}\right)
$$

In order to have a stable scheme, we must bound the amplitude of the error $\varepsilon_{j}^{k}$ for any $(j, k)$ such that

$$
|\nu| \leq 1
$$

From the expression C.1 of $\nu$, we can show that

$$
1-r \delta t-2 \frac{\delta t}{\delta B^{2}} \leq \nu \leq 1
$$

Therefore, the scheme is stable when the condition 4.5) is satisfied.

\section{Additional results for Section 3}

\section{D.1 Another PDE for the basket derivative price}

It is noteworthy to mention that the PDE 3.8 can be reformulated as follows:

$$
\frac{\partial V^{c}}{\partial t}+\frac{1}{2}\left(\sigma^{c}\right)^{2} \frac{\partial^{2} V^{c}}{\partial\left(S^{c}\right)^{2}}+r S^{c} \frac{\partial V^{c}}{\partial S^{c}}-r V^{c}=0
$$

with the final condition $V^{c}\left(T, S^{c}\right)=H\left(S^{c}(T)\right)$, whereas the boundary conditions are obtained by investigating the situations $S^{c}=0$ and $S^{c} \rightarrow+\infty$. The technical aspects discussed above for pathdependent options and American-type options have also to be taken into account in a similar way when solving the PDE (D.1).

The differential equation (D.1) is similar to the classical, two-dimensional, Black-Scholes partial differential equation in the univariate case. Moreover, the different terms in the Equation (D.1) have a clear interpretation. The first term deals with the 'Theta' of the derivative, i.e. the time decay. The term containing the second derivative considers the convexity, the so-called 'Gamma' of the derivative, and together with the 'Theta', they represent the dispersion part of the equation. The convection part is given by the 'Delta', or first derivative, and makes the portfolio risk-free. Finally, the ' $-r V^{c}$ ' term is the reaction, which is due to the discounting. Note that the coefficients in front of the Delta and the Gamma are time dependent, whereas the coefficients of the PDE (3.8) are all constant. Although we do not report them here, numerical tests confirm that due to the time dependency of the coefficients in (D.1), the computation time to solve (3.8) is lower. 


\section{D.2 On the distribution of the baskets}

Theorem 3.2 provides a solution to the differential equation as an integration over the future states of the Brownian motion with drift $B_{\lambda}$. A simple trapezoidal rule or Gauss quadrature can be employed to find an accurate estimate. In general, the price of a multi-asset derivative in a non-comonotonic market is an $n$-dimensional integral which is hard to numerically implement. In this subsection, we recast the solution for the European-type derivative $V_{\lambda}^{c}$ in terms of future states of the comonotonic basket $S^{c}$. As a result, we obtain a closed-form pdf for the comonotonic basket from which an approximate pdf of the original basket is derived. We first state the following lemma.

Lemma D.1 For any $x \in \mathbb{R}_{+}$, there exists a value $F_{S^{c}}(x ; T, t) \in(0,1)$ satisfying the equation:

$$
\sum_{i=1}^{n} w_{i} S_{i}^{c}(t) \mathrm{e}^{\left(r-\frac{1}{2} \sigma_{i}^{2}\right)(T-t)+\sigma_{i} \sqrt{T-t} \Phi^{-1}\left(F_{S^{c}}(x ; T, t)\right)}=x,
$$

where $\Phi$ is the cdf of a standard normal distribution. Moreover, $F_{S^{c}}(x ; T, t)$ is the time-t risk-neutral cdf of $S^{c}(T)$ evaluated in $x$ :

$$
F_{S^{c}}(x ; T, t)=\mathbb{Q}\left[S^{c}(T) \leq x \mid \mathcal{F}_{t}\right]
$$

Now we express the European-type comonotonic basket derivative price $V^{c}$ in function of the future realizations of the comonotonic basket $S^{c}$ at time $T$ and provide a closed form expression for the pdf of $S^{c}$. We use the notation $V^{c}\left(t, S^{c}\right)$ for the price at time $t$.

Theorem D.1 The price at time t of a European-type derivative with pay-off function $H$ written on the comonotonic sum $S^{c}$ is denoted by $V^{c}\left(t, S^{c}\right)$ and given by

$$
V^{c}\left(t, S^{c}\right)=\mathrm{e}^{-r(T-t)} \int_{0}^{+\infty} H(s) f_{S^{c}}(s ; T, t) \mathrm{d} s,
$$

where

$$
f_{S^{c}}(s, T, t)=\frac{\phi_{T-t}\left(\sqrt{T-t} \Phi^{-1}\left(F_{S^{c}}(s ; T, t)\right)\right)}{\sum_{i=1}^{n} \sigma_{i} w_{i} S_{i}^{c}(t) \mathrm{e}^{\left(r-\frac{1}{2} \sigma_{i}^{2}\right)(T-t)+\sigma_{i} \sqrt{T-t} \Phi^{-1}\left(F_{S^{c}}(s ; T, t)\right)}},
$$

is the time-t risk-neutral density of the comonotonic basket $S^{c}(T)$.

Expression (D.4) then states that the price $V^{c}\left(t, S^{c}\right)$ can be found by determining the pay-off $H(s)$, multiplying this future pay-off with the risk-neutral comonotonic basket density and finally discounting the future expected pay-off.

\section{D.3 Proof of Lemma D.1}

Assume that we are at time $t$, i.e. the time- $t$ prices $S_{i}^{c}(t)$ are known for $i=1,2, \ldots, n$. We can use Expression 3.12) to rewrite the comonotonic basket price $S^{c}(T)$ as follows:

$$
S^{c}(T)=\sum_{i=1}^{n} w_{i} S_{i}^{c}(t) \mathrm{e}^{\left(r-\frac{1}{2} \sigma_{i}^{2}\right)(T-t)+\sigma_{i}\left(B_{\lambda}(T)-B_{\lambda}(t)\right)} .
$$


Under the risk-neutral measure $\mathbb{Q}$, we find $B_{\lambda}(T)-B_{\lambda}(t) \stackrel{d}{=} \sqrt{T-t} \Phi^{-1}(U)$, where $U$ is uniformly distributed and $\Phi$ is the cdf of a standard normal distribution. Hence, we have:

$$
S^{c}(T) \stackrel{d}{=} \sum_{i=1}^{n} w_{i} S_{i}^{c}(t) \mathrm{e}^{\left(r-\frac{1}{2} \sigma_{i}^{2}\right)(T-t)+\sigma_{i} \sqrt{T-t} \Phi^{-1}(U)} .
$$

Denote by $F_{S^{c}}(x ; T, t)$ the time- $t$ cdf of $S^{c}(T)$ under the risk-neutral probability measure:

$$
F_{S^{c}}(x ; T, t)=\mathbb{Q}\left[S^{c}(T) \leq x \mid \mathcal{F}_{t}\right] .
$$

Using the additivity property of the inverse cdf for comonotonic sums (see Theorem 6 in Dhaene et al. (2002a)), we find:

$$
F_{S^{c}}^{-1}(p ; T, t)=\sum_{i=1}^{n} w_{i} S_{i}^{c}(t) \mathrm{e}^{\left(r-\frac{1}{2} \sigma_{i}^{2}\right)(T-t)+\sigma_{i} \sqrt{T-t} \Phi^{-1}(p)} .
$$

We end the proof by noticing that the $\operatorname{cdf} F_{S^{c}}$ is strictly increasing and continuous, and hence:

$$
F_{S^{c}}^{-1}\left(F_{S^{c}}(x ; T, t) ; T, t\right)=x .
$$

\section{D.4 Proof of Theorem D.1}

Recall from Theorem 3.2 that the solution $V^{c}$ can be expressed as follows:

$$
V^{c}\left(t, S^{c}\right)=\mathrm{e}^{-r(T-t)} \int_{-\infty}^{+\infty} H\left(\sum_{i=1}^{n} w_{i} S_{i}^{c}(t) \mathrm{e}^{\left(r-\frac{1}{2} \sigma_{i}^{2}\right)(T-t)+\sigma_{i} y}\right) \phi_{T-t}(y) \mathrm{d} y .
$$

We switch from the variable $y$ to the new variable $s$, where

$$
s=\sum_{i=1}^{n} w_{i} S_{i}^{c}(t) \mathrm{e}^{\left(r-\frac{1}{2} \sigma_{i}^{2}\right)(T-t)+\sigma_{i} y} .
$$

Using Lemma D.1, we find:

$$
y=\sqrt{T-t} \Phi^{-1}\left(F_{S^{c}}(s ; T, t)\right),
$$

which leads to:

$$
\mathrm{d} s=\left(\sum_{i=1}^{n} \sigma_{i} w_{i} S_{i}^{c}(t) \mathrm{e}^{\left(r-\frac{1}{2} \sigma_{i}^{2}\right)(T-t)+\sigma_{i} \sqrt{T-t} \Phi^{-1}\left(F_{S^{c}}(s ; T, t)\right)}\right) \mathrm{d} y .
$$

The price $V^{c}\left(t, S^{c}\right)$ can then be expressed as (D.4).

In order to prove that $f_{S^{c}}$ is the risk-neutral density function of the comonotonic basket, we have to prove that this function is positive, tends to zero at the limits and integrates to one. Moreover, it has to correspond with the risk-neutral cdf $F_{S^{c}}(s, T, t)$. Putting $H(s)=1$ in (D.4) shows that the area below the function $f_{S^{c}}$ is equal to one. Moreover, one easily verifies that the function is positive and $f_{S^{c}}(s, T, t) \rightarrow 0$ if $s \rightarrow 0$ and $s \rightarrow+\infty$. This proves that $f_{S^{c}}$ is a valid density function. Consider the pay-off function $H(z)=\mathbb{I}(s \leq x)$. The price $V^{c}$ of the corresponding derivative can now be expressed as in (D.4), which results in $\int_{0}^{x} f_{S^{c}}(s, T, t) \mathrm{d} s=F_{S^{c}}(x, T, t)$. We can now conclude that $f_{S^{c}}$ is the risk-neutral pdf of the comonotonic basket. 